\title{
TOPIES IN MULTIPLE-LOOP REGULATORS AND CURRENT-MODE PROGRAMMING
}

\author{
R. D. MIDDLEBROOK \\ Californio Institute of Technology \\ Pasodena, Californio
}

\section{ABSTRACT}

Some general considerations about multiple-loop beedback are discussed, and it is concluded that incorporation of a current-programmed power stage into a "new" power stage model is both justified and useful. A new circuit-oriented model of the current feedback path is derived which augments the well-known power stage canonical circuit model.

The current loop gain, though wideband, is always stable if the conventional stabilizing ramp is employed, but has a relatively small lowfrequency value. Consequently, the "new" power stage is more usefully modelled by a $y$ parameter model in which the current loop is not explicit. Expressions for the $y$ parameters are given that are extensions of those previously derived.

Although current-programming tends to make the power stage output behave as a current source, the control to output voltage transfer function exhibits, in addition to the familiar dominant pole, a second pole at the current loop gain crossover frequency, which may lie from one-sixth to twothirds of the switching frequency.

\section{INTRODUCTION}

Current-programming has become widely adopted as a useful technique for easing the design and improving the performance of switched-mode regulators. The basic principle and the resulting advantages of current-programming were discussed in two papers at PESC $1978[1,2]$.

A sma11-signal model was presented at PESC 1979 [3]. A current-programmed power stage inside the conventional output voltage feedback loop constitutes an example of multiple-loop feedback, and the approach taken in [3] was to find a general model of the current-programmed power stage so that its relevant transfer functions could be used as

This work was conducted under the Power Electronics Program supported by grants from the General

Telephone Company and Emerson Electric Company, and under contracts from IBM Tucson and San Jose, and the McDonnel1 Douglas Astronautics Company. factors in the remaining output voltage loop gain. Thus, the "minor" current-programmed feedback loop was absorbed into a "new" power stage model so that design of the "major," or overa11, output voltage feedback loop could proceed on the conventional single-1oop basis.

At PESC 1984 the claim was made [4] that the loop gain of this major output voltage feedback loop does not provide meaningful information on the stability or the dynamics of the regulator system, and that the "proper" loop for study is the one that is "inside" all of the identified loops.

In the presence of multiple loops, the simple concepts related to single-loop feedback systems become fuzzy, and the first objective of this paper is to try to clarify some of these concepts in the multiple-loop case. Actually, all the useful properties of a linear system, such as transfer functions, stability, and sensitivities, can be defined and determined without "loops" ever being mentioned. The trouble with this formal approach is that insight into the physical system is sacrificed, which makes design difficult in spite of the existence of the analysis. On the contrary, one would like to retain and extend the simple singleloop concepts as far as possible, and to this end one is at liberty to identify any number of loops one wishes. The objective, of course, is to establish a model that retains as many of the distinct features of the physical system, and at the same time lends itself to useful and simple analysis. This is the essence of "design-oriented analysis." The discussion of multiple-loop systems in Section 2 leads to a reiteration of the modelling objective stated in [3], namely, that absorption of a current-mode loop into a "new" power stage mode1 inside the output voltage major loop is both legitimate and useful.

A second objective of this paper is to extend the equivalent circuit model of the "new" currentmode programmed power stage, and to derive it by a circuit-oriented method which is both analogous to and an extension of that by which the original canonical model of the duty ratio programmed power stage was derived [5]. In [3], the "new" currentprogrammed power stage model was derived by formal 
application of the state-space averaging method [6], and the y parameter equivalent circuit model, in which the current-feedback loop is no longer visible, emerged full-blown at the end, perhaps leaving the reader uncertain as to how or why it happened. In contrast, the derivation presented here attempts to retain as much physical significance and motivation as possible, both for the duty-ratio and current-programmed modes.

One new result of this approach is that a current-programmed loop gain can be quantitatively defined. It turns out that this loop is always stable (as long as the well-known stabilizing ramp is employed), contains yet another "minor" voltage loop in the case of the buck converter, and may have a rather low gain. All of these results are hardly surprising, since absence of knowledge about them has not prevented rapid increase in the successful implementation of current-programming. These results also lend weight to the argument that the current-programmed loop is not worth identifying and should be absorbed into the "new" power stage model.

The basic objective of current-programming is to make the power stage into a current source feeding the output capacitance and load. The input also, incidentally, approaches a constant current. Since duty ratio programmed discontinuous conduction mode has the same effects [7], it is not surprising that both modes are suitably represented by a y parameter model. Moreover, quantitatively, the "conduction parameter" $\mathrm{K}$ introduced to characterize the discontinuous conduction mode also plays an integral role in the current-programmed model.

Some additional aspects of the currentprogrammed model are considered in Section 5 . The $y$ parameter model apparent1y has no resemblance to the original canonical model for the duty ratio programmed mode, and so in Section 5.1 an attempt is made to manipulate the output part of the $y$ parameter model into the original LC filter format. This shows that current-programming introduces a series damping resistance and reduces the effective inductance value, which is why the output approximates a current source. Comparison is made with storage-time modulation in the power switch, which was shown in [8] to lead to exactly the same result.

Current-programming, which may be considered an extreme case of storage-time modulation, causes sufficient damping that the two poles of the LC filter become real and wel1-separated so that the lower one becomes dominant, corresponding to the we11-known "single-pole response." However, an important result of the model derived here is that the second pole is still present, and may lie at one-sixth to two-thirds of the switching frequency. This two-pole filter transfer function enters into the control-to-output transfer function, which is derived in Section 5.2. The presence of the second pole may therefore have a significant effect on the phase margin of the regulator major voltage feedback loop.

\section{MULTIPLE-LOOP FEEDBACK}

Any function of a linear system of constant lumped elements, such as its gain $G$, can be expressed as the ratio of a numerator $N(s)$ to a demoninator $\mathrm{D}(\mathrm{s})$, each of which is a polynomial in complex frequency $s$ :

$$
G(s)=\frac{N(s)}{D(s)}
$$

The seminal work on the properties of such systems was done by Bode [9]. The roots of the numerator are the zeros of the function, and the roots of the denominator are the poles of the system which are the natural (unforced) frequencies, and are the same regardless of what function $G$ is being represented.

The system is stable if no natural frequency has a positive real part, that is, if no system pole lies in the right half-plane ( $r h p)$. The Routh criterion [10] is a test for determining if there are any rhp poles.

Stability against oscillation is a necessary but not sufficient condition for acceptability of a system design. One also needs to know how various functions of the system, for example its output impedance, depend upon certain element values and, in particular, how such functions vary when the element values change. To find this, one can derive the numerator and denominator of the function each into two groups such that one group, $\mathrm{N}_{1}(\mathrm{~s})$ and $\mathrm{D}_{1}(\mathrm{~s})$, does not contain a certain element $\mathrm{W}_{\mathrm{k}}$ and the other group, $\mathrm{N}_{2}(\mathrm{~s})$ and $\mathrm{D}_{2}(\mathrm{~s})$, is proportional to $\mathrm{W}_{\mathrm{k}}$ and so can be written $\mathrm{N}_{2}(\mathrm{~s})=\mathrm{W}_{\mathrm{k}} \mathrm{N}_{\mathrm{k}}(\mathrm{s})$ and $D_{2}(s)=W_{k} D_{k}(s)$, so that

$$
G(s)=\frac{N_{1}(s)+N_{2}(s)}{D_{1}(s)+D_{2}(s)}=\frac{N_{1}(s)+W_{k} N_{k}(s)}{D_{1}(s)+W_{k} D_{k}(s)}
$$

This involves merely arranging $G(s)$ as a bilinear function of $W_{k}$, and this can always be done since it is easily shown that $W_{k}$ cannot appear in the numerator or in the denominator to any power higher than the first [11], although one or more of the $N^{\prime} s$ and D's in (2) may be zero. Hence, $W_{k}$ is explicit in (2) and, by differentiation,

$$
\frac{\Delta G}{G}=\left(\frac{1}{1+D_{2} / D_{1}}-\frac{1}{1+N_{2} / N_{1}}\right) \frac{\Delta W_{k}}{W_{k}}
$$

This gives the fractional change in $G$ that results from a given fractional change in $W_{k}$, that is, the sensitivity of the function $G$ to changes in a given element $W_{k}$.

The stability of a system, and the dependence of various functions upon certain elements, and their sensitivities, are all that one needs to know about a system. Significantly, in the above summary, no mention has been made of "loops" or, indeed, of whether any "feedback" is present or not. "Feedback loops" are conveniences in modelling a system identified so that constituents of various 
functions can be associated with certain physical properties.

For example, suppose that a system gain $G$ is written as

$$
\begin{aligned}
G=\frac{N}{D} & =\frac{N}{D_{p}+D_{q}} \\
& =\frac{N / D_{q}}{1+\left(N / D_{q}\right)\left(D_{p} / N\right)} \equiv \frac{G_{1}}{1+G_{1} H_{1}}
\end{aligned}
$$

The final form is that obtained from the ideal block diagram of a single-loop feedback system whose forward gain is $G_{1}$ and feedback path gain is $H_{1}$, as

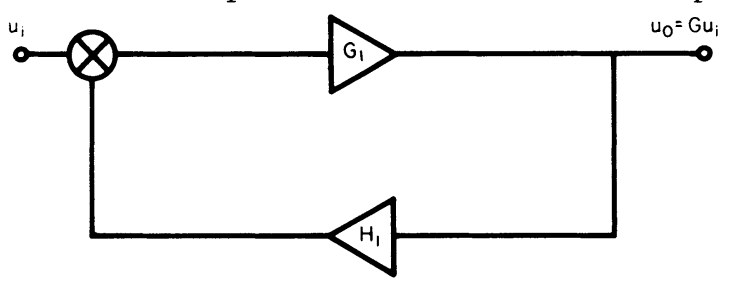

Fig. 1. A single-loop feedback system.

in Fig. 1. The product $\mathrm{G}_{1} \mathrm{H}_{1}$ can be defined as the loop gain $\mathrm{T}_{1}$, so that $\mathrm{G}$ can also be written

$$
G=\frac{N / D_{q}}{1+D_{p} / D_{q}}=\frac{G_{1}}{1+T_{1}}
$$

Stability can now be examined by means of the Nyquist criterion [10], which states that for stability a polar plot of the loop gain $\mathrm{T}_{1}$ must encircle the critical point $(-1,0)$ counter-clockwise as many times as the number of rhp poles of $\mathrm{T}_{1}$. In the simplest case, this number is zero.

The usefulness of form (5) or (6) lies in the identification of $G_{1}$ and $H_{1}$ with separate blocks in the model of the physical system, and $\mathrm{T}_{1}$ as a loop connection of these blocks. If this can be done, yet another form of (5) exposes one of the fundamental properties of feedback:

$$
G=\frac{1}{H_{1}} \quad \frac{G_{1} H_{1}}{1+G_{1} H_{1}}=\frac{1}{H_{1}} \frac{T_{1}}{1+T_{1}}
$$

This form says that the closed-1oop gain approaches the reciprocal of the feedback path gain if the loop gain is sufficiently large, and that the sensitivity of $G$ to variations in any element to which $\mathrm{T}_{1}$ is proportional is reduced by $1+\mathrm{T}_{1}$ from its open-loop value. These, and other we11-known properties of single-loop feedback, are true only if every element in the system is inside either $G_{1}$ or $\mathrm{H}_{1}$, but not both.

There are two disadvantages of forms (5), (6), or (7). First, there are two requirements for stability that need to be examined: whether or not $\mathrm{T}_{1}$ has rhp poles, and then whether or not $\mathrm{T}_{1}$ satisfies the Nyquist criterion. In the simplest case, the system is "open-loop stable" and $\mathrm{T}_{1}$ does not have rhp poles; then, the Nyquist criterion can be replaced by the test for gain and phase margins on a Bode plot of $\mathrm{T}_{1}$.

The other disadvantage is that not all of the system elements appear as a proportional factor in $\mathrm{T}_{1}$. This is because the particular $\mathrm{T}_{1}=\mathrm{D}_{\mathrm{p}} / \mathrm{D}_{\mathrm{q}}$ that corresponds to the physical loop may not be defined in terms of the $D_{p}$ and $D_{q}$ that correspond to $D_{1}$ and $\mathrm{D}_{2}$ of $\mathrm{Eq}$. (2), that is, a certain element $\mathrm{W}_{\mathrm{k}}$ in general appears in both $\mathrm{D}_{\mathrm{p}}$ and $\mathrm{D}_{\mathrm{q}}$ so that the sensitivity of the gain to that element is not necessarily determined by $\mathrm{T}_{1}$. However, there is a $\mathrm{T}_{\mathrm{k}}=\mathrm{D}_{2} / \mathrm{D}_{1}$ that is proportional to $\mathrm{W}_{\mathrm{k}}$, so there are at least as many "loop gains" as there are elements in the system, although some of them may be the same. Actually, there are more "loop gains" than this because the system denominator $\mathrm{D}$ can be divided quite arbitrarily into a $D_{p}$ and $a D_{q}$, regardless of how any element enters into one or both.

Thus there are an infinite number of loop gains, each with a gain and phase margin, and each of which must satisfy the Nyquist criterion for stability. In general the margins are different, although they all converge to zero as the system approaches instability.

The function $G_{1}=N / D_{q}$ in $E q .(5)$ is also a ratio of two polynomials in $\mathrm{s}$, and its denominator can also be separated into two groups, so that $G_{1}$ can be expressed as a function of two new parameters $G_{2}$ and $H_{2}$. Also, a factor $G_{3}$ can arbitrarily be taken out of the original $\mathrm{N}$, so that $\mathrm{G}_{1}$ can be written

$$
G_{1}=G_{3} \frac{G_{2}}{1+G_{2} H_{2}}
$$

Hence, the original $G$ can be expressed as

$$
G=\frac{G_{3} \frac{G_{2}}{1+G_{2} H_{2}}}{1+G_{3} \frac{G_{2}}{1+G_{2} H_{2}} H_{1}}
$$

This is the form that would be obtained from a system block diagram that has a minor loop $\mathrm{T}_{2}=\mathrm{G}_{2} \mathrm{H}_{2}$ inside the major loop $\mathrm{T}_{1}=\mathrm{G}_{1} \mathrm{H}_{1}$, as in Fig. 2 .

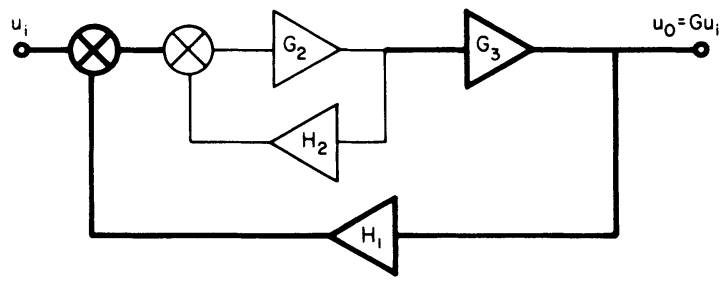

Fig. 2. A two-loop feedback system.

Stability of the system in Fig. 2 can be examined in the same way as before: the gain $G_{2}$ is examined for rhp poles, and then the Nyquist criterion is applied to $\mathrm{T}_{2}$. The procedure is then repeated for $G_{1}$ and $T_{1}$. This process can obviously be extended to any number of minor loops [12]. 
The question of which is the "right" loop gain with its gain and phase margins is therefore meaningless. The proper question is, which loop gain or gains are useful for the designer to examine? Obviously, those whose component blocks correspond to the physical system blocks, in spite of the fact that such correspondence is not always possible exactly. One may take advantage of what correspondence there is, otherwise one may as well ignore the concept of loops altogether.

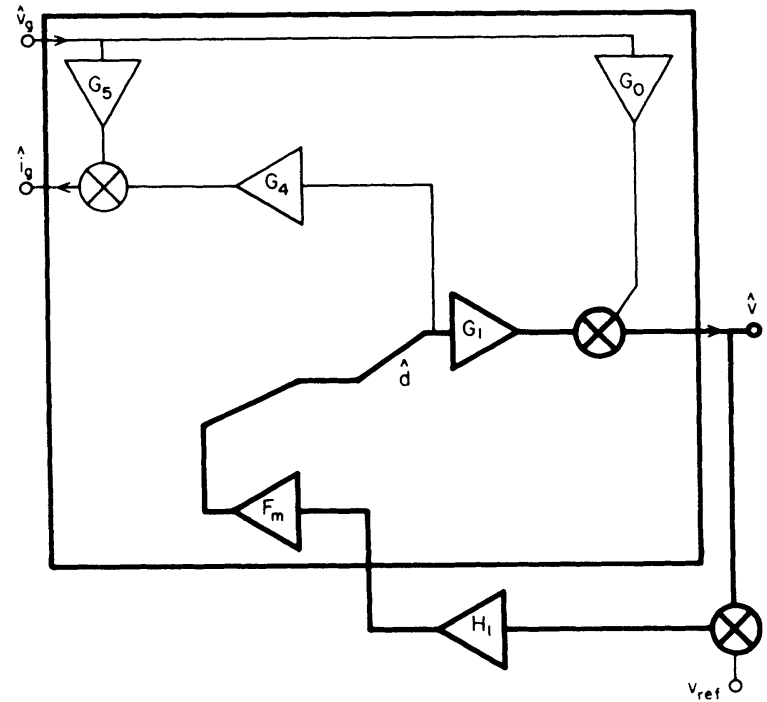

Fig. 3. Block diagram of a single-loop voltage regulator.

To illustrate this process consider the system of Fig. 3, which represents the small-signal properties of a switched-mode regulator. The $\mathrm{H}_{1}$ box is the error amplifier, the $\mathrm{F}_{\mathrm{m}}$ box is the modulator that converts the analog control signal $\hat{v}_{c}$ into the duty ratio $\hat{d}$, and the $G_{1}$ box determines the output voltage $\hat{v}$. The single feedback loop is closed through the summing junction that compares the output voltage with the reference. The line voltage $\hat{v}_{g}$ also affects the output voltage through the $G_{0}$ box. The line current $\hat{i}_{g}$ is determined by both $\hat{d}$ and $\hat{v}_{g}$ through the $\mathrm{G}_{4}$ and $\mathrm{G}_{5}$ boxes. All of the boxes inside the large box except the modulator $\mathrm{F}_{\mathrm{m}}$ constitute the model for the power stage.

Figure 4 shows the block diagram of a voltage regulator that includes a current-programmed power stage. The diagram has extra gain blocks, $G_{2}$ to identify the inductor current that is being programmed, and $\mathrm{H}_{2}$ to represent the current that is fed back to determine the duty ratio. An extra gain box $\mathrm{G}_{3}$ indicates that the line input $\hat{v}_{\mathrm{g}}$ affects the inductor current directly and, as will be seen later, $\hat{v}_{g}$ also directly affects the duty ratio, so another gain box $\mathrm{G}_{6}$ is necessary.

Not only is there now a minor current loop $\mathrm{F}_{\mathrm{m}} \mathrm{G}_{2} \mathrm{H}_{2}$ but, as also will be seen later, in the case of the buck converter there is yet another loop $\mathrm{G}_{1} \mathrm{H}_{3}$ closed via $\mathrm{H}_{3}$. This occurs because in a buck converter the inductor charging ramp, and hence the duty ratio, is determined by the output voltage as well as by the line input voltage. The diagram of Fig. 4 contains that of Fig. 2 in [4], and corresponding blocks have the same symbol.
It is claimed in [4] that the only "proper" place to determine loop gain and stability margins is at $\mathrm{T}_{1}$ in Fig. 4, apparently on the grounds that this is the only point at which all loops come together. However, this is not necessarily true, because the error amplifier $\mathrm{H}_{1}$ may itself contain a local feedback loop. This very commonly occurs, and in fact is present in the experimental circuit described in [4].

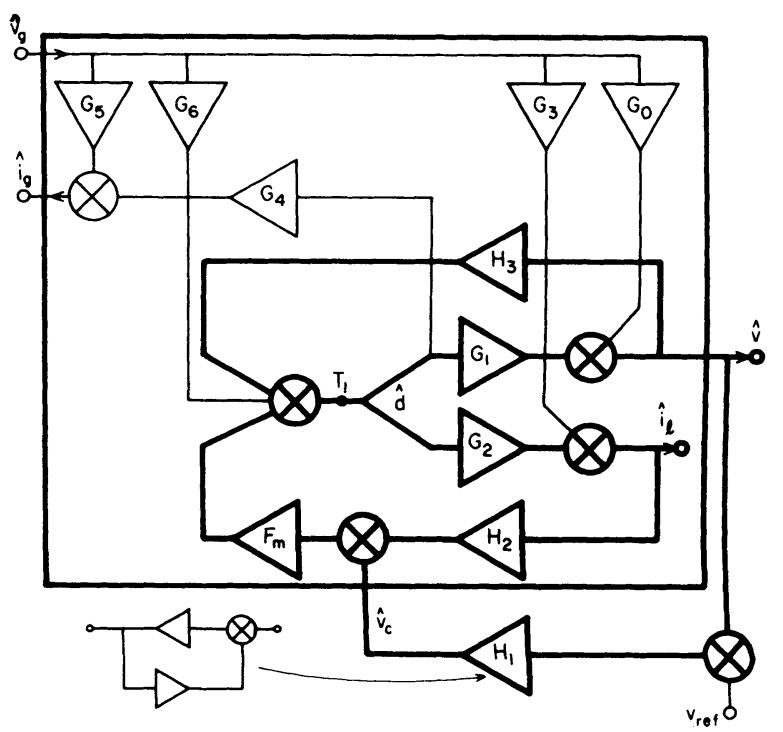

Fig. 4. Block diagram of a multiple-loop voltage regulator, including a current-programmed loop.

Feedback loops in general are of two types: those that are intentionally installed in order to regulate some particular quantity, and those that are unintentional and arise from some inherent property of a device or circuit configuration. In the current-programmed regulator represented by Fig. 4, three of the loops are intentional: the minor loop in the $\mathrm{H}_{1}$ error amplifier, the currentprogramming loop $\mathrm{F}_{\mathrm{m}} \mathrm{G}_{2} \mathrm{H}_{2}$, and the major voltage 1oop; the minor loop $\mathrm{G}_{1} \mathrm{H}_{3}$ is unintentional.

Since for analysis purposes identification of loops is arbitrary, it seems eminently reasonable to choose the intentional loops and to consider them sequentially, that is, to treat the innermost "nested" loop first, find its closed-loop properties, then use those in treatment of the next loop, and so on [12]. The only possible difficulty arises when a minor loop is unstable, in which case stability of the next outer loop must be considered on a more elaborate basis, as discussed in [4]. However, this situation rarely occurs in practical power supplies.

This procedure emphasizes the quantities that the intentional loops were put there to regulate, and gives the most help in design. It contains no inherent approximations, and is an entirely correct procedure that will be followed in the later sections on modelling the current-programmed loop. 


\section{DERIVATION OF EQUIVALENT CIRCUIT MODELS}

The state-space averaging technique has been applied to modelling dc-to-dc converter power stages in duty ratio programmed continuous conduction mode [6], duty ratio programmed discontinuous conduction mode [7], and in current-programmed continuous conduction mode [3]. In [6] and [7], alternative circuit-oriented derivations were also presented, which have the advantage of retaining insight into the physical operation of circuit. The principal purpose of this section is to present a corresponding circuit-oriented derivation of the model for current-programmed continuous conduction mode.

Current-programming consists of a local feedback loop closed around a converter operating in the duty ratio programmed mode, usually in continuous conduction. The approach, therefore, is to find a model of the local feedback path that can be added to the already established model of the converter. This is done in Section 3.2, after the derivation of the duty ratio programmed model is reviewed in Section 3.1.

\subsection{Model for Duty Ratio Programming}

The key to a simple model of a switched-mode $\mathrm{dc}-\mathrm{to}-\mathrm{dc}$ converter is the averaging out of the switching frequency, which is accomplished by replacement of the single-pole double-throw switch by a transformer having a conversion ratio equal to an appropriate duty ratio of the switch.

The basic representations of the buck, boost, and buck-boost converters are shown in Fig. 5, as established in [6] for the continuous conduction mode. The buck-boost converter is shown with a noninverted output to facilitate comparison with the models of the other two. The straight and wavy lines through the transformer symbol are a reminder that the conversion ratio is valid for dc as well as ac. The large-signal, time-dependent line input and output voltages are $v_{g}$ and $v$, the input and output currents are $i_{g}$ and $i$ (the capacitor $C$ is counted as part of the load), and the inductor current is il. The switch duty ratio is d, referenced to the inductor energy storing fraction of the switching cycle, also time dependent, and $\mathrm{d}^{\prime}$ is its complement $1-\mathrm{d}$.

The models of Fig. 5 are sufficient to solve for the linear line to output transfer function, but are nonlinear for variations in duty ratio $d$. The models are linearized by adoption of a small-signal constraint. Each large-signal, time-varying quantity is replaced by a large-signal dc component and a superimposed small-signal ac component, $\mathrm{v}=\mathrm{V}+\hat{\mathrm{v}}, \mathrm{d}=\mathrm{D}+\hat{\mathrm{d}}$, etc.

The buck-boost model will be discussed as an example. The first step is the replacement of the transformer by an equivalent pair of generators, as in Fig. 6(a), which applies to the d':l transformer of either the boost or the buck-boost converter. Each generator is divided into its components, and those proportional to $D^{\prime}$ are restored into a trans-
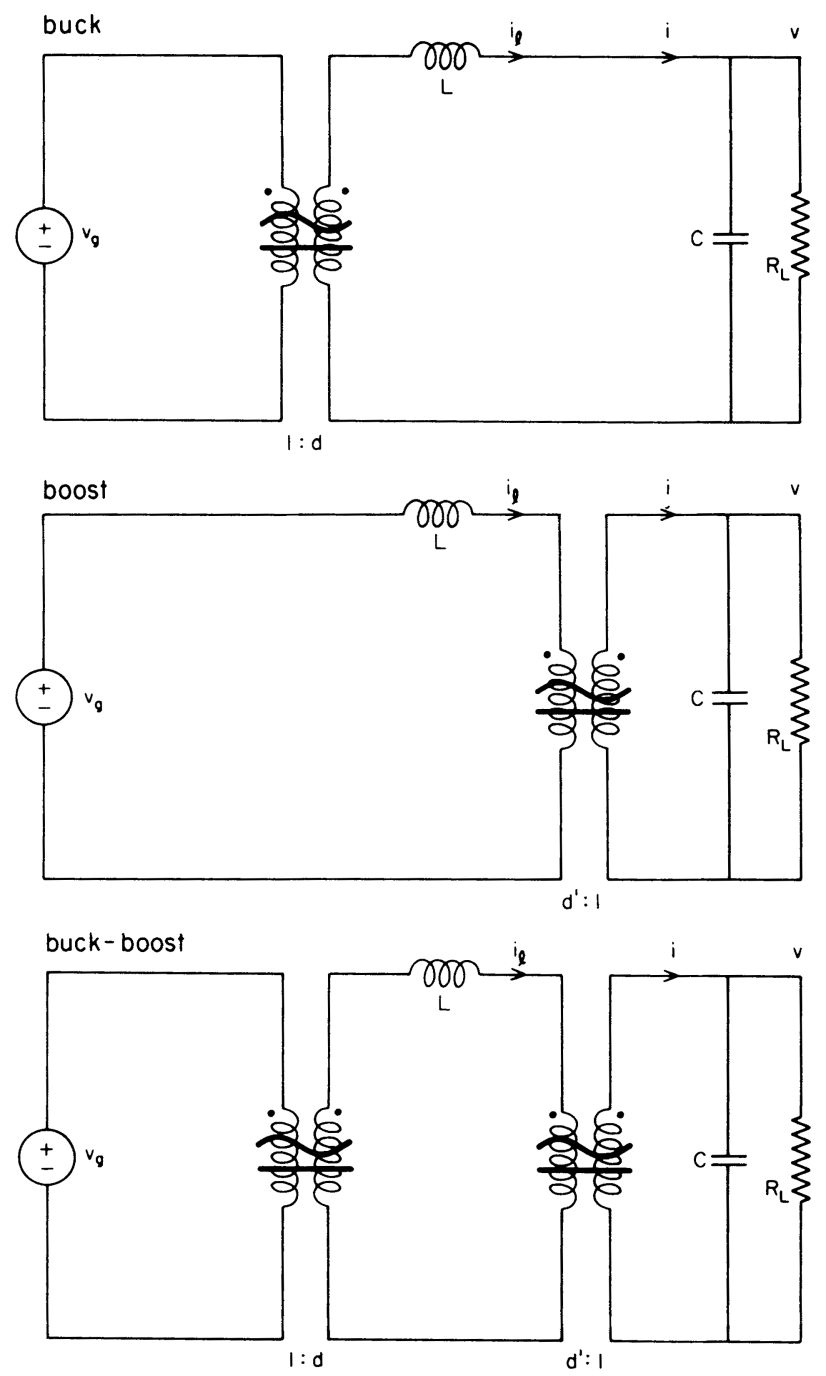

Fig. 5. Basic state-space averaged models of the buck, boost, and buck-boost converters.

former with a constant conversion ratio $D^{\prime}: 1$. Those proportional to $\hat{d}$ are separated into independent generators, and the remaining components in the products of ac quantities are eliminated subject to the small-signal restriction.

The result of these steps is shown in Fig. 6(b), which also includes substitutions for Il. First, the $\mathrm{dc}$ inductor current $\mathrm{I}_{\ell}$ is equal to $1 / \mathrm{D}^{\prime}$ times the $\mathrm{dc}$ converter output current $\mathrm{I}$. Second, $\mathrm{I}=\mathrm{V} / \mathrm{R}$ where $\mathrm{V}$ is the converter dc output voltage and $R$ is defined as

$$
R \equiv \frac{V}{I}=\frac{d c \text { output voltage }}{d c \text { output current }}
$$

Note that $R=R_{L}$ if the converter dc load is $R_{L}$, but $R$ is not necessarily equal to $R_{L}$ if, for example, the converter has a constant-current load component, or if the load $R_{L}$ is a negative resistance such as the input of a switched-mode regulator. That is, $R$ represents the output dc operating point of the converter regardless of the nature of its load, and 
a)

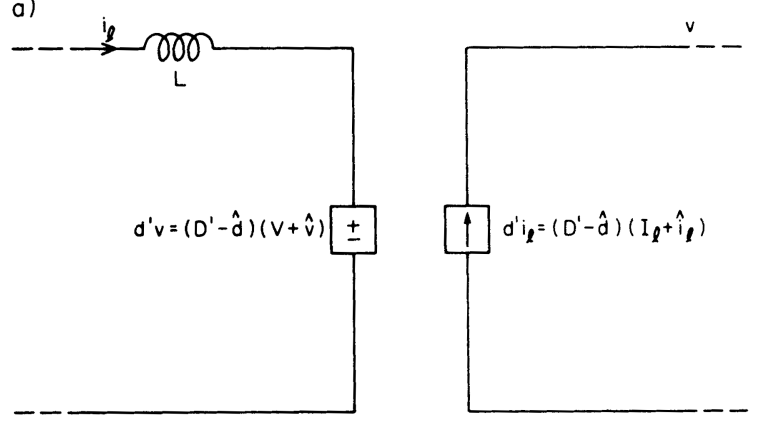

e)

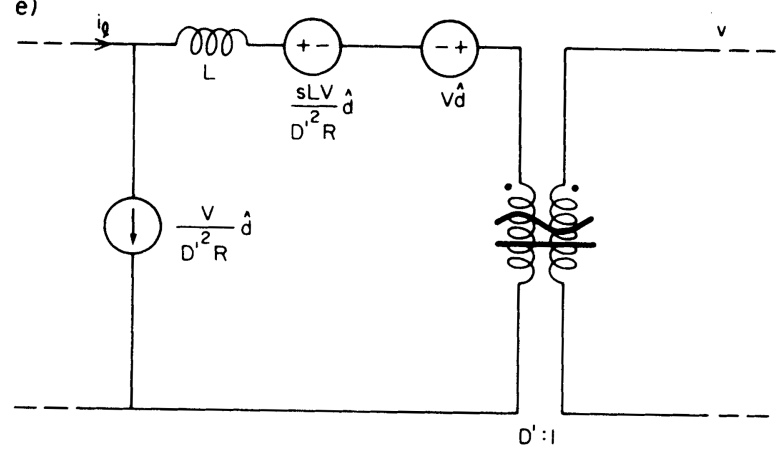

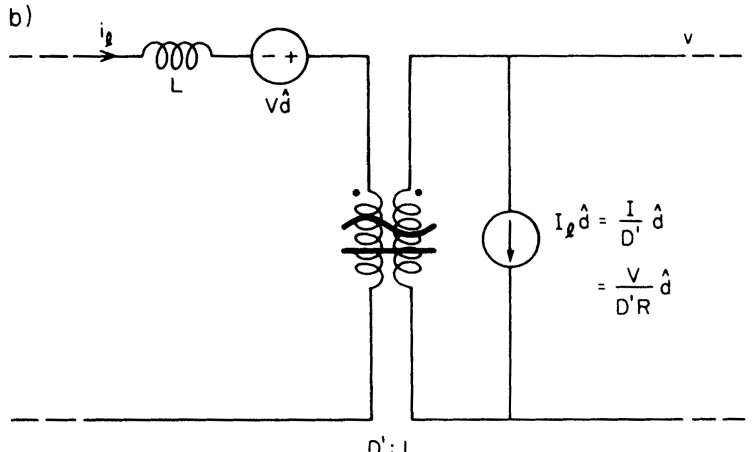

c)

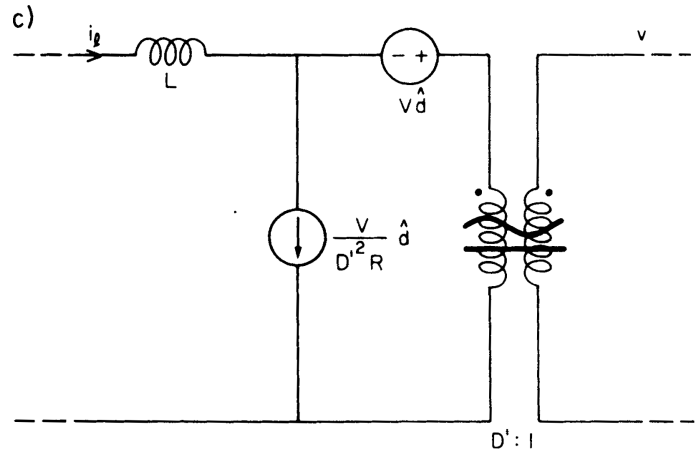

d)

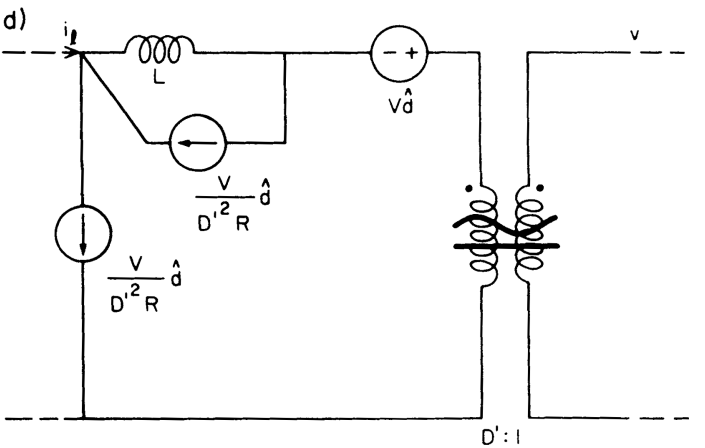

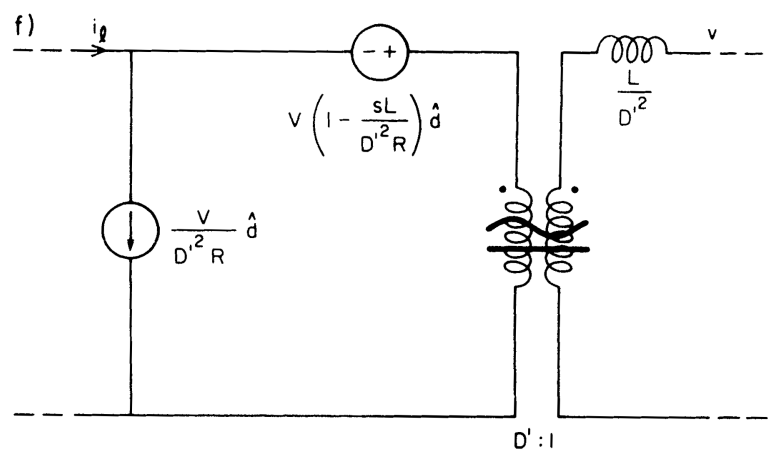

Fig. 6. Steps in the emergence of the rhp zero in the model of the boost or the buck-boost converter.

may be called the "output operating point parameter."

In the next step, Fig. 6(c), the current generator is reflected to the transformer primary, toward the objective of moving the generators to the left of the model and the inductance to the right. Interchange of the current generator and the inductance can be accomplished in the two steps of Figs. $6(\mathrm{~d})$ and $6(\mathrm{e})$ : first, the current generator is separated into two equal generators in series, and their junction connected to the other side of the inductance. The Norton equivalent formed by the parallel generator and the inductance is then converted into the Thevenin series equivalent. Note that the effect of these steps is to move the current generator "through" the inductance, with the appearance of a new voltage generator that accounts for the drop in the inductive reactance due to the current generator. The two voltage generators can then be combined as in Fig. 6(f), and the inductance is at the same time passed through the voltage generator and reflected to the transformer secondary.

The same process can be performed on the other transformer of the buck-boost converter, and the complete model to this stage is shown in Fig. 7(a). In Fig. 7(b), all the generators are moved to the left, and the voltage generators are combined. The two current generators could also be combined, and indeed this is the last step taken in the original derivation of the "canonical model" [6], in which the "modulation" generators are at the left, the 

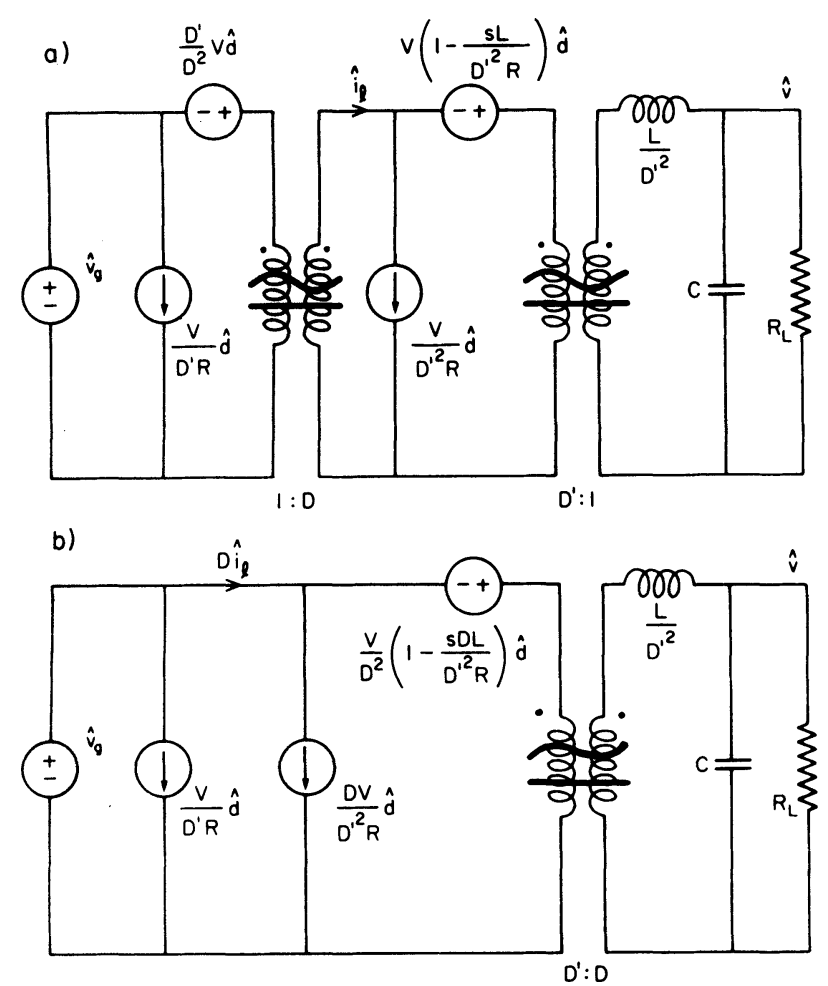

Fig. 7. Two further steps in the development of the canonical model for the buck-boost converter in duty ratio programmed mode.

"conversion transformer" is in the middle, and the "effective filter" is at the right.

Here, however, this last step is not taken, because the next objective is to consider currentprogramming in which the inductor current is sensed and so must not be lost from the model. With this in mind, the inductor current $i_{\ell}$ was identified in each of the successive manipulations of Fig. 6, in spite of the departure of the "effective" inductance $\mathrm{L} / \mathrm{D}^{\prime} 2$ from the location where if exists. In Fig. 7 (b), il is still identified but as Dil, the current on the other side of the 1:D transformer; but, this is as far as one can go in reduction of the model, because combining the two current generators would cause the branch in which Dil is identified to be lost.

It may be noted that the rhp zero factor $\left(1-s D L / D^{12} R\right)$ in the voltage modulation generator arises because the power switch separates the $L$ and $C$, and in the model derivation the current modulation generator is moved "through" the inductance. A similar result occurs for the boost converter, but not for the buck because the power switch is already at the left of both the $\mathrm{L}$ and the $\mathrm{C}$.

\subsection{Mode1 for Current-Programming}

In duty ratio programming, the duty ratio of the power switch is directly determined by the control voltage $v_{c}$ in the modulator, which is usually a simple comparator. The block diagram of the modulator and power stage is shown in Fig. 8.

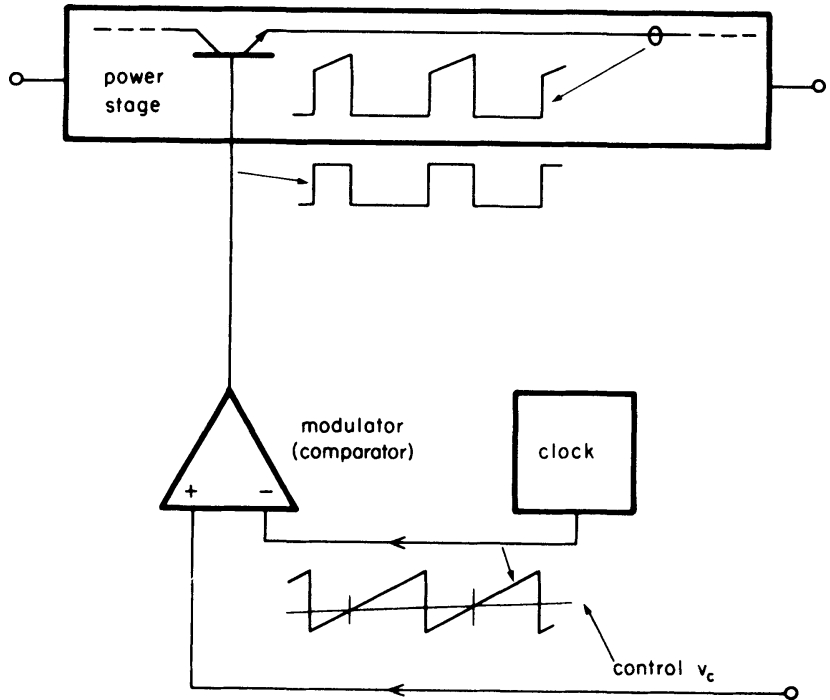

Fig. 8. Functional relationships in the modulator and power stage under duty ratio programming.

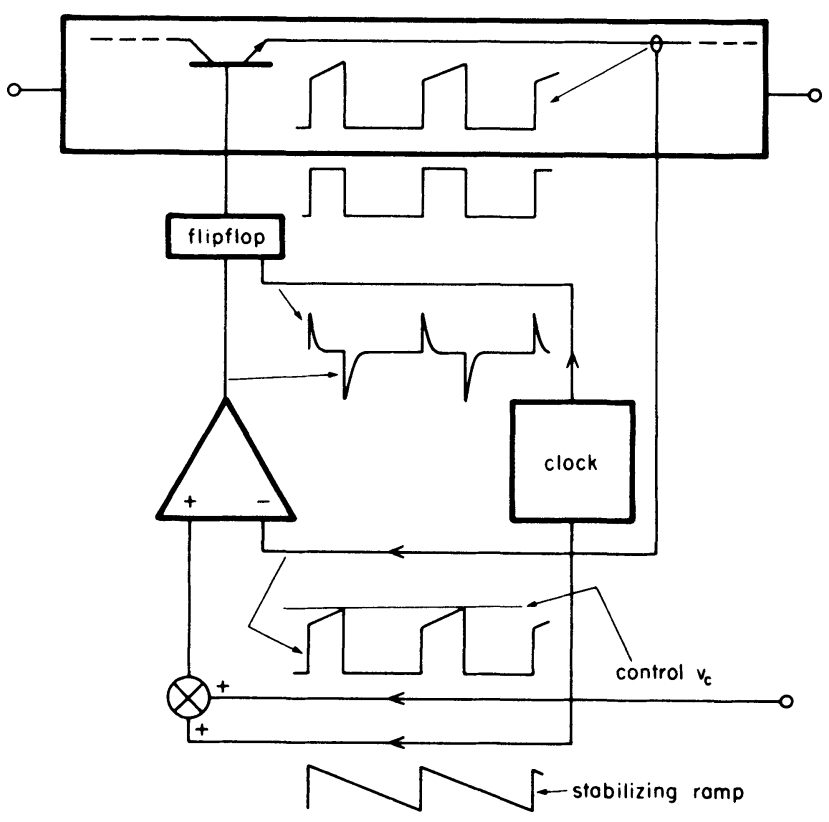

Fig. 9. Functional relationships in the modulator and power stage under current-programming.

The clock signal, in the form of a voltage ramp, is applied to one comparator input, and turns the power switch oN uniformly at the clock, or switching, frequency $f_{S}=1 / T_{S}$. The ON-time is terminated after an interval $\mathrm{dT}_{\mathrm{s}}$ when the ramp overtakes the control signal $v_{c}$ applied to the other comparator input. Since the ramp is linear, the duty ratio is determined as

$$
\mathrm{d}=\frac{\mathrm{v}_{\mathrm{c}}}{\mathrm{v}_{\mathrm{m}}}
$$


where $V_{m}$ is the height of the ramp. This relation can be divided into $\mathrm{dc}$ and ac parts:

$$
D=\frac{v_{c}}{v_{m}} \quad \hat{d}=\frac{\hat{v}_{c}}{v_{m}}
$$

These relations represent the transfer function of the modulator, and are used in conjunction with the canonical model of the power stage to determine the control to output and line to output transfer functions, and any other function of interest.

In current-programming, shown in Fig. 9, the clock as before initiates the switch ON-time uniformly at the frequency $\mathrm{f}_{\mathrm{S}}$. However, the clock signal applied to the comparator is now replaced by a voltage proportional to the current flowing in the power stage inductor, which is also a ramp. Thus, the switch ON-time is terminated when the inductor current reaches a value proportional to the control signal, where the proportionality factor $R_{f}$ is the ratio between the voltage presented to the comparator and the current ramp that produces it. Clearly, a physical "current" feedback loop exists.

As discussed elsewhere $[1,3]$ an inherent instability exists in this current feedback loop for duty ratios greater than 0.5 , unless a stabilizing ramp is introduced. This principle is also illustrated in Fig. 9, in which a negative current ramp, also derived from the clock, produces a proportional voltage that is added to the control signal. For convenience, the same proportionality factor $R_{f}$ is employed as for the inductor current.

Thus, in current-programming mode, the switch duty ratio is not uniquely determined by the control signal, but by how long it takes the inductor current to reach the value determined by the control signa1. The essence of the modelling objective is therefore to find the relationship between the duty ratio and the quantities that determine it, in a form compatible with the averaging technique already employed for the power stage.

The relationship is determined at the comparator input, whose detailed waveforms over one switch$v_{c} / R_{f}$

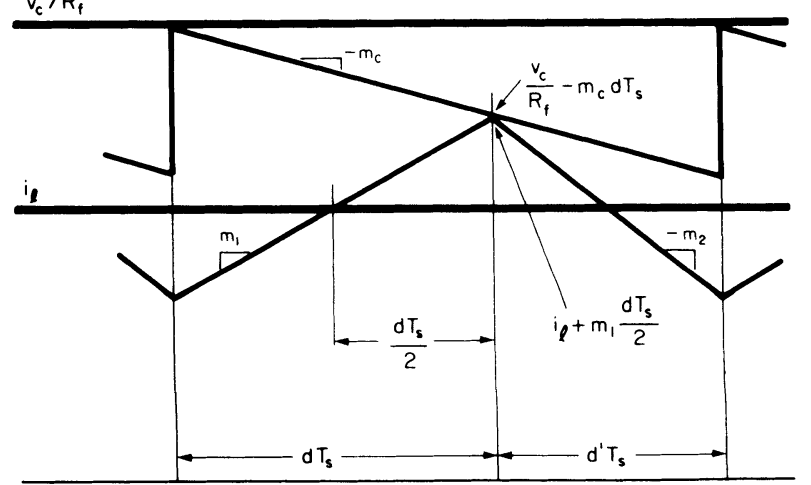

Fig. 10. Waveforms at the modulator input in current-programmed mode: the duty ratio is determined by intersection of the inductor current ramp with the control voltage plus stabilizing ramp. ing period $\mathrm{T}_{\mathrm{S}}$ are shown in Fig. 10. The voltage waveforms are scaled to equivalent current waveforms by the proportionality factor $R_{f}$. One comparator input carries the control current $v_{c} / R_{f}$ plus the negative stabilizing current ramp of slope $-\mathrm{m}_{\mathrm{C}}$; the other input carries the inductor current with average value il. However, the instantaneous inductor current has a positive ramp of slope $t_{1}$ when the power switch is $\mathrm{ON}$ and a negative ramp of slope $-m_{2}$ when the switch is OFF, and the duty ratio $d$ is determined by intersection of the two comparator inputs which, from the geometry of the waveform diagram, is given by

$$
i_{\ell}+m_{1} \frac{d T}{2}=\frac{v_{c}}{R_{f}}-m_{c} d T_{s}
$$

It remains only to find the dc and small-signal ac solutions for $d=D+\hat{d}$. As in the power stage modelling procedure, we set $i_{\ell}=I_{\ell}+\hat{i}_{\ell}$ and $\mathrm{v}_{\mathrm{c}}=\mathrm{v}_{\mathrm{c}}+\hat{\mathrm{v}}_{\mathrm{c}}$, and also $\mathrm{m}_{1}=\mathrm{M}_{1}+\hat{\mathrm{m}}_{1}$ because the inductor ramp slope is affected by the voltage across it which also has $\mathrm{dc}$ and ac components. On the other hand, we set $m_{c}=M_{c}$ because usually the stabilizing ramp is constant.

The $d c$ and ac solutions of (13) are

$$
\begin{aligned}
& \mathrm{D}=\frac{2}{\mathrm{nM}_{1} \mathrm{~T}_{s}}\left(\frac{\mathrm{V}_{\mathrm{c}}}{\mathrm{R}_{\mathrm{f}}}-\mathrm{I}_{\ell}\right) \\
& \hat{\mathrm{d}}=\frac{2}{\mathrm{nM}_{1} \mathrm{~T}_{s}}\left(\frac{\hat{\mathrm{v}}_{\mathrm{c}}}{\mathrm{R}_{\mathrm{f}}}-\hat{\mathrm{i}}_{\ell}\right)-\frac{\mathrm{D}}{\mathrm{nM}_{1}} \hat{\mathrm{m}}_{1}
\end{aligned}
$$

where

$$
\mathrm{n} \equiv 1+\frac{2 \mathrm{M}_{\mathrm{c}}}{\mathrm{M}_{1}}
$$

Products of ac quantities are ignored according to the usual linearizing small-signal restriction.

All the above equations apply to any converter. The differences between converters result solely from how the inductor ramp $\mathrm{m}_{1}$ is dependent upon operating conditions.

\section{Buck Converter}

During the switch ON-time the inductor is connected between line input and output voltages $\mathrm{v}_{\mathrm{g}}$ and $\mathrm{v}$, so

$$
\mathrm{m}_{1}=\frac{\mathrm{v}_{\mathrm{g}}-\mathrm{v}}{\mathrm{L}}
$$

The dc conversion ratio is $\mathrm{V}=\mathrm{DV}$, hence

$$
M_{1}=\frac{D^{\prime} v}{D L} \text { and } \hat{m}_{1}=\frac{\hat{v}_{g}-\hat{v}}{L}
$$

Substitution into (15) gives

$$
\hat{\mathrm{d}}=\frac{\mathrm{KRD}}{\mathrm{nD^{ \prime } v}}\left(\frac{\hat{\mathrm{v}}_{\mathrm{c}}}{\mathrm{R}_{\mathrm{f}}}-\hat{i}_{\ell}\right)-\frac{\mathrm{D}^{2}}{\mathrm{nD}^{\prime} \mathrm{v}} \hat{\mathrm{v}}_{\mathrm{g}}+\frac{\mathrm{D}^{2}}{\mathrm{nD}^{\prime} \mathrm{v}} \hat{\mathrm{v}}
$$


where

$$
\mathrm{K} \equiv \frac{2 \mathrm{~L}}{\mathrm{RT}_{\mathrm{S}}}
$$

\section{Boost Converter}

During the switch ON-time the inductor is connected across the line input voltage $\mathrm{v}_{\mathrm{g}}$, so

$$
\mathrm{m}_{1}=\frac{\mathrm{v} g}{\mathrm{~L}}
$$

The dc conversion ratio is $V=V_{g} / D^{\prime}$, hence

$$
M_{1}=\frac{D^{\prime} V}{L} \text { and } \quad \hat{m}_{1}=\frac{\hat{v}_{g}}{L}
$$

which leads to

$$
\hat{\mathrm{d}}=\frac{\mathrm{KR}}{\mathrm{nD^{ \prime } \mathrm { V }}}\left(\frac{\hat{\mathrm{v}}_{\mathrm{c}}}{\mathrm{R}_{\mathrm{f}}}-\hat{\mathrm{i}}_{\ell}\right)-\frac{\mathrm{D}}{\mathrm{nD^{ \prime } \mathrm { v }}} \hat{\mathrm{v}}_{\mathrm{g}}
$$

Buck-boost Converter

As for the boost converter,

$$
m_{1}=\frac{v g}{L}
$$

However, the dc conversion ratio is $\mathrm{V}=\mathrm{DV}_{\mathrm{g}} / \mathrm{D}^{\prime}$, hence

$$
M_{1}=\frac{D^{\prime} V}{D L} \text { and } \quad \hat{m}_{1}=\frac{\hat{v}_{g}}{L}
$$

which leads to

$$
\hat{\mathrm{d}}=\frac{\mathrm{DKR}}{\mathrm{nD^{ \prime } V}}\left(\frac{\hat{v}_{\mathrm{c}}}{\mathrm{R}_{\mathrm{f}}}-\hat{i}_{\ell}\right)-\frac{\mathrm{D}^{2}}{\mathrm{nD^{ \prime } V}} \hat{\mathrm{v}}_{\mathrm{g}}
$$

The parameter $\mathrm{n}$ is a function of the stabilizing ramp $M_{c}$, by (16). If $M_{c}=M_{2}$, the negative slope of the inductor current during the switch OFF-time $\mathrm{D}^{\prime} \mathrm{T}_{S}$, which is a common choice, then $\mathrm{n}=1+2 \mathrm{M}_{2} / \mathrm{M}_{1}$. It is obvious from the geometry of Fig. 10 that $M_{2} / M_{1}=D / D^{\prime}$ so that, for $M_{C}=M_{2}$,

$$
\mathrm{n}=1+\frac{2 \mathrm{D}}{\mathrm{D}^{\prime}}=\frac{1+\mathrm{D}}{\mathrm{D}^{\prime}}=\frac{1+\mathrm{D}}{1-\mathrm{D}}
$$

Therefore, for all three converters, $\mathrm{n}$ is between unity for zero stabilizing ramp and (1+D)/D' for a stabilizing ramp with $\mathrm{M}_{\mathrm{C}}=\mathrm{M}_{2}$.

Equations (19), (23), and (26) show how the ac duty ratio $\hat{d}$ is determined by not only the control voltage $\hat{v}_{c}$ but also by the inductor current $\hat{i}_{\ell}$ and, in general, also by the converter line input and output voltages $\hat{v}_{g}$ and $\hat{v}_{\text {. The coefficients }}$ of the various contributing terms can be represented by gain blocks connected between appropriate points in the canonical models of the three converters already discussed. The results are shown in Figs. 11 through 13 . The circles representing the modu- lation generators are replaced by squares to indicate that they are now dependent upon signals elsewhere in the same system.

The current feedback loop identified in the general current-programmed mode configuration of Fig. 9 is evident in all three specific equivalent circuits of Figs. 11 through 13. However, there is also a voltage loop in the model of the buck converter of Fig. 11; this occurs because the inductor current ramp during the switch oN-time is a function of the output voltage $\hat{v}$ as well as of the line input voltage $\hat{v}_{g}$.

It is now clear why the two current modulation generators in the buck-boost model were not combined: the sensed inductor current $i_{\ell}$ must be identified, and since it appears in the model as Dil the extra factor $D$ must be cancelled by the additional $1 / D$ gain box in the equivalent circuit of Fig. 13. For comparison with the block diagram of Fig. 4, the gain boxes in Figs. 11 through 13 are also labelled with the corresponding symbols.

\section{$\frac{\text { 4. PROPERTIES OF THE CURRENT-PROGRAMMED }}{\text { POWER STAGE }}$}

The equivalent circuit models of Figs. 11 through 13 contain a11 the information necessary to determine various transfer functions. However, since there is at least one physical feedback loop present in each model, it is of interest to determine appropriate loop gains and to see whether the simple concepts of feedback illuminate the system properties. This is done in Section 4.1, and it is concluded that the current loop gain is not large. Therefore, in Section 4.2, an overall model is derived in which the current loop is absorbed and no longer identified.

\subsection{Current Loop Gain $\mathrm{T}_{\mathrm{C}}$}

Because the buck converter model exhibits two feedback loops, it will be considered last.

\section{Boost Converter}

To determine the current loop gain $\mathrm{T}_{c}$ for the boost converter, the control signal $\hat{v}_{c}$ and the line input voltage $\hat{v}_{g}$ are set to zero, and a current $\hat{i}_{z}$ is injected at a suitable point in the loop, as shown in the corresponding reduced model in Fig. 14.

The loop gain is $T_{c}=\hat{i}_{y} / \hat{i}_{x}=\hat{i}_{\ell} / \hat{i}_{x}$, where the sensed current $\hat{i}_{\ell}$ can be found as the sum of the currents $\hat{i}_{a}$ and $\hat{i}_{b}$ identified in Fig. 14. Hence

$$
T_{c}=\frac{\hat{i}_{a}+\hat{i}_{b}}{\hat{i}_{x}}=F_{m}\left(\frac{V\left(1-s L / D^{\prime 2} R\right)}{D^{\prime 2}\left(Z_{L}+s L / D^{\prime 2}\right)}+\frac{V}{D^{\prime 2} R}\right)
$$

which reduces to

$$
T_{c}=\frac{K}{n D^{\prime 3}} \frac{z_{L}+R}{z_{L}+s L / D^{\prime 2}}
$$




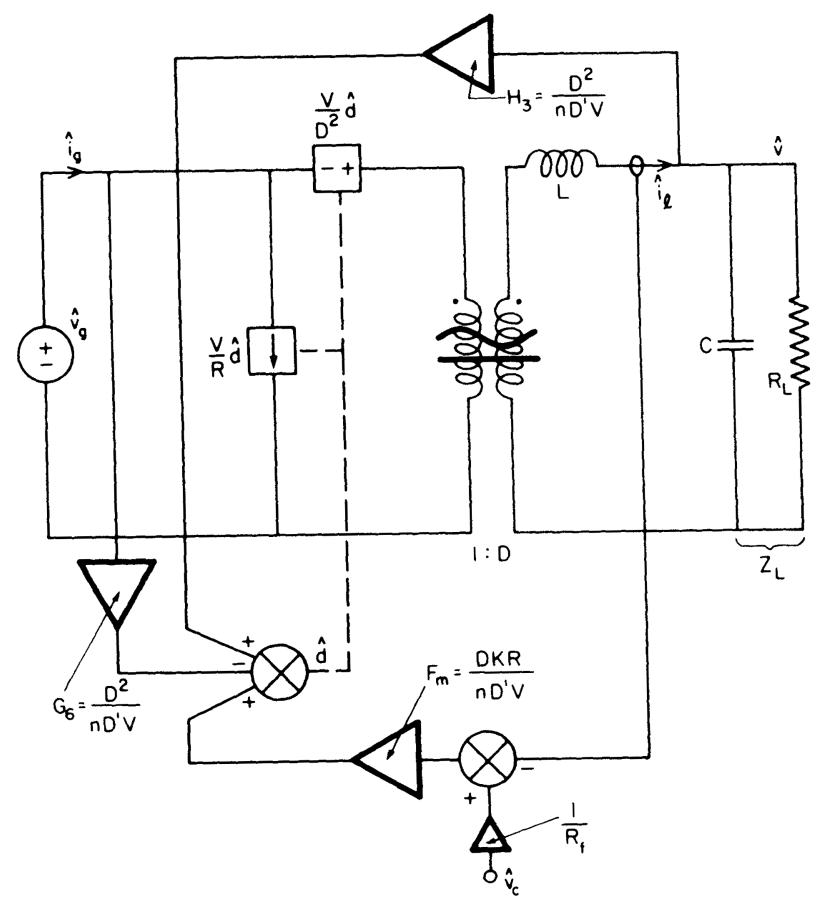

Fig. 11. Basic model of the current-programmed buck converter, showing explicitly the current loop via $F_{m}$ closed around the canonical model for the duty ratio programmed mode. There is an inner voltage loop via $\mathrm{H}_{3}$.

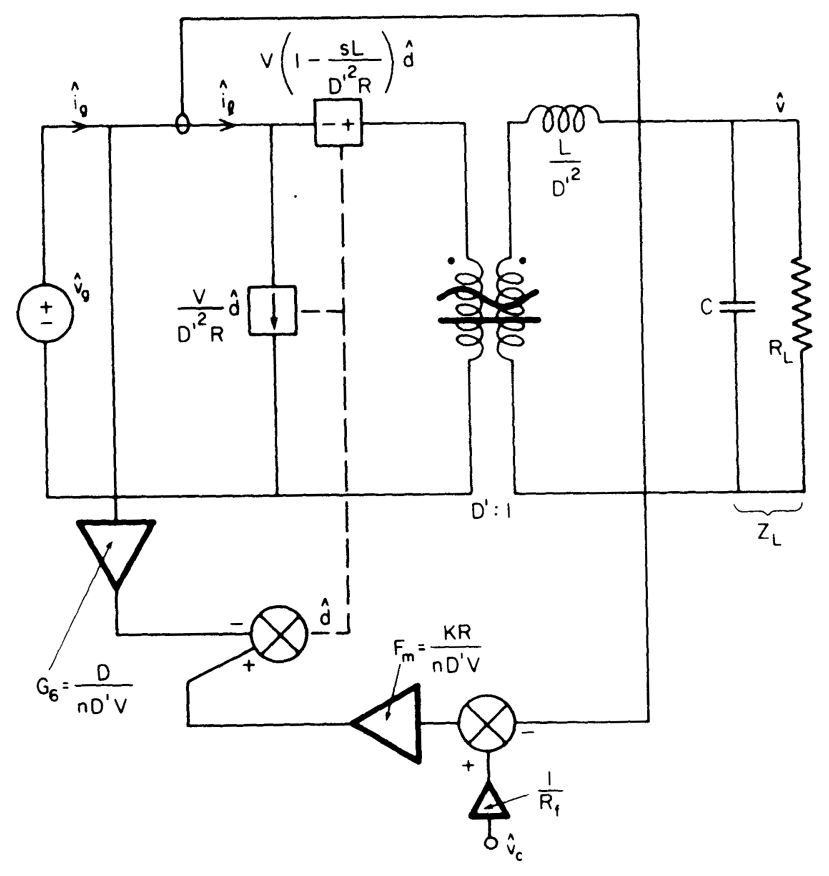

Fig. 12. Basic model of the current-programmed boost converter, showing the current loop via $F_{m}$ closed around the canonical model for the duty ratio programmed mode.

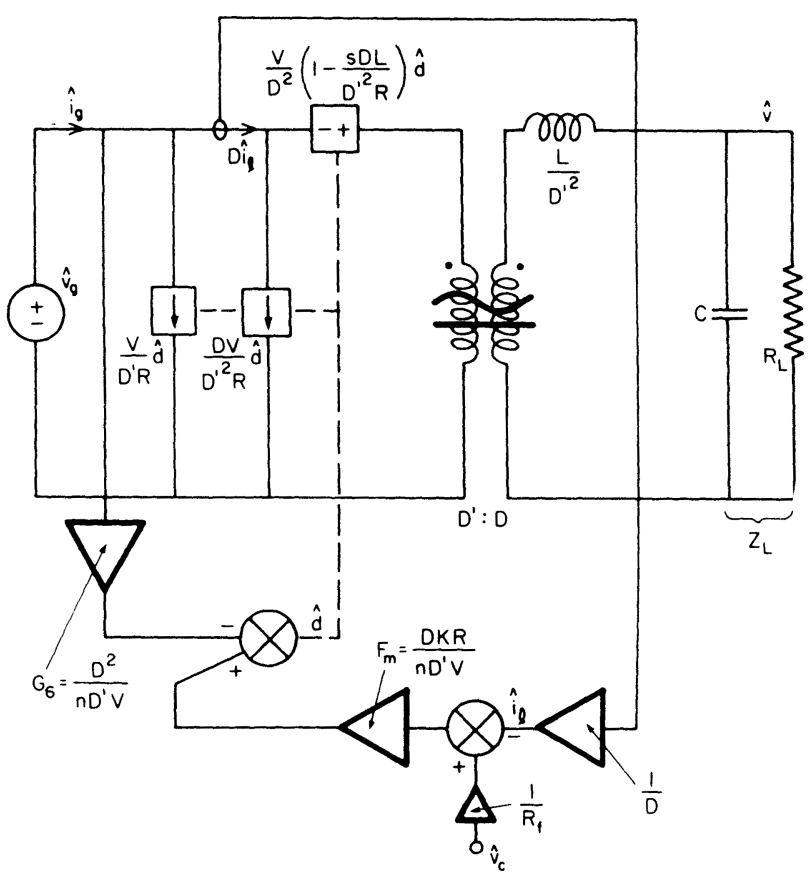

Fig. 13. Basic model of the current-programmed buck-boost converter, showing the current loop via $F_{m}$ closed around the canonical model for the duty ratio programmed mode.

With $\mathrm{Z}_{\mathrm{L}}=\mathrm{R}_{\mathrm{L}} / /(1 / \mathrm{sC})$, this becomes

$$
T_{C}=\frac{K}{n D^{\prime 3}}\left(1+\frac{R}{R_{L}}\right) \frac{1+s\left(R_{L} \| R\right) C}{1+\frac{1}{Q_{e}}\left(\frac{s}{\sqrt{D^{\prime 2} / L C}}\right)+\left(\frac{s}{\sqrt{D^{\prime 2} / L C}}\right)^{2}}
$$

where $Q_{e} \equiv R_{L} / \sqrt{L / D^{\prime 2} C}$, but may be considered generalized to include damping sources other than the load resistance $R_{L}$.

Buck-boost Converter

The model is similar to that of Fig. 14 except for differences in the way that $D$ enters into some of the factors. The result is

$$
\begin{aligned}
\mathrm{T}_{\mathrm{C}} & =\frac{\mathrm{DK}}{\mathrm{nD}^{\prime 3}} \frac{\mathrm{Z}_{\mathrm{L}}+\mathrm{R} / \mathrm{D}}{\mathrm{Z}_{\mathrm{L}}+\mathrm{sL} / \mathrm{D}^{\prime 2}} \\
& =\frac{\mathrm{DK}}{\mathrm{nD}^{\prime 3}}\left(1+\frac{\mathrm{R}}{\mathrm{DR}}\right) \frac{1+\mathrm{s}\left[\mathrm{R}_{\mathrm{L}} / /(\mathrm{R} / \mathrm{D})\right] \mathrm{C}}{1+\frac{1}{\mathrm{Q}_{\mathrm{e}}}\left(\frac{\mathrm{s}}{\sqrt{\mathrm{D}^{\prime 2}} / \mathrm{LC}}\right)+\left(\frac{\mathrm{s}}{\sqrt{\mathrm{D}^{\prime 2} / \mathrm{LC}}}\right)^{2}}
\end{aligned}
$$

Buck Converter

The reduced model for calculation of $T_{c}$ is shown in Fig. 15. There is now an inner voltage loop, whose loop gain $\mathrm{T}_{\mathrm{v}}$ is easily seen to be 


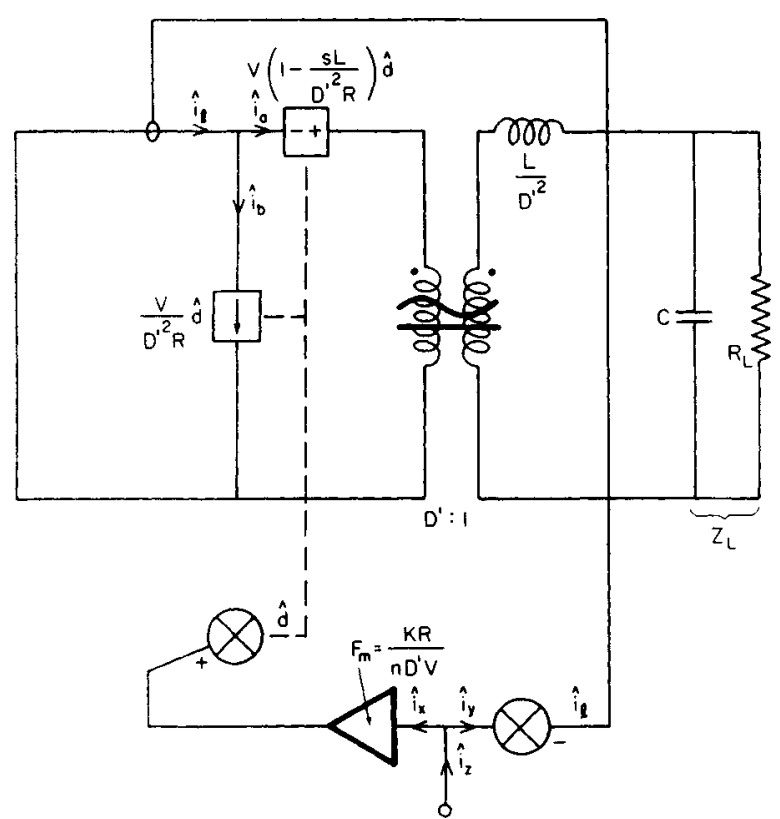

Fig. 14. Reduced model of the boost converter of Fig. 12, for calculation of the current loop gain $T_{c}=\hat{i}_{y} / \hat{i}_{x}$ by injection of $a$ current $\hat{i}_{z}$.

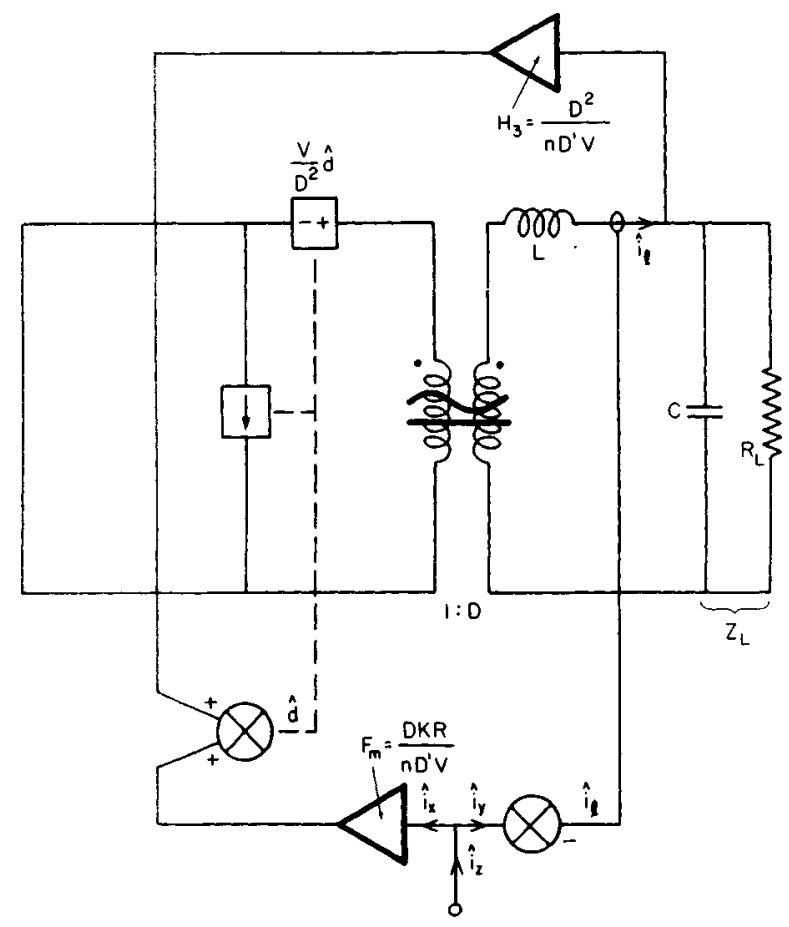

Fig. 15. Reduced model of the buck converter of Fig. 11, for calculation of the current loop gain $T_{c}=\hat{\imath}_{y} / \hat{\imath}_{x}$ by injection of $a$ current $i_{z}$.

$$
T_{v}=\frac{V}{D^{2}} D_{e} \frac{D^{2}}{n D^{\prime} v}=\frac{D / D^{\prime}}{n} H_{e}
$$

where $\mathrm{H}_{\mathrm{e}}=\mathrm{Z}_{\mathrm{L}} /\left(\mathrm{Z}_{\mathrm{L}}+\mathrm{sL}\right)$ is the effective voltage transfer function of the loaded output filter. It is to be noted that this is a positive loop gain, so that $\hat{d}=F_{m} \hat{i}_{x} /\left(1-T_{v}\right)$ instead of $\hat{d}=F_{m} \hat{i}_{x}$ as it would have been in the absence of the voltage loop.

In accordance with the sequential procedure for examining nested loops advocated in Section 2, we may determine the stability of the voltage loop itself by examining $1-\mathrm{T}_{\mathrm{V}}$ for rhp roots. The result is that (unless $R_{L}$ is negative) $1-T_{v}$ does not have rhp roots if $D^{\prime} D^{\prime}<n$. However, this is the same condition required for the stability of the current loop as a whole, from which the value of the stabilizing ramp is determined. To see this, consider the requirement on the stabilizing ramp slope $M_{C}$ for current loop stability [3]:

$$
\frac{M_{2}-M_{c}}{M_{1}+M_{c}}<1
$$

This is equivalent to

$$
\frac{M_{2}}{M_{1}}<1+\frac{2 M_{c}}{M_{1}}
$$

which is the same as

$$
\frac{\mathrm{D}}{\mathrm{D}^{\top}}<\mathrm{n}
$$

Therefore, it may be concluded that choice of the stabilizing ramp to meet the conventional criterion also ensures stability of the inner voltage loop, and therefore precludes the presence of any rhp poles in the current loop gain $\mathrm{T}_{\mathrm{C}}$, which can then be found from Fig. 15 as

$$
\begin{aligned}
T_{c} & =\frac{\hat{i}_{y}}{\hat{i}_{x}}=\frac{\hat{i}_{\ell}}{\hat{i}_{x}}=\frac{V}{D^{2}} D \frac{1}{z_{L}+s L} \frac{F_{m}}{1-T_{v}} \\
& =\frac{K}{n^{\prime} D_{n}} \frac{R}{z_{L}+s L / D_{n}} \\
& =\frac{K}{n D^{\prime} D_{n}} \frac{R}{1+\frac{1}{Q_{L}}\left(\frac{s}{\sqrt{D_{n} / L C}}\right)+\left(\frac{s}{\sqrt{D_{n} / L C}}\right)^{2}}
\end{aligned}
$$

where

$$
\mathrm{D}_{\mathrm{n}} \equiv 1-\mathrm{D} / \mathrm{nD}^{\prime}
$$

The magnitude asymptote constructions for the loop gain $\mathrm{T}_{\mathrm{C}}$ for the three converters from Eqs. (30), (32), and (39) are shown in Fig. 16. The actual values at the apexes, not shown, depend on the particular values of $Q_{e}$. 


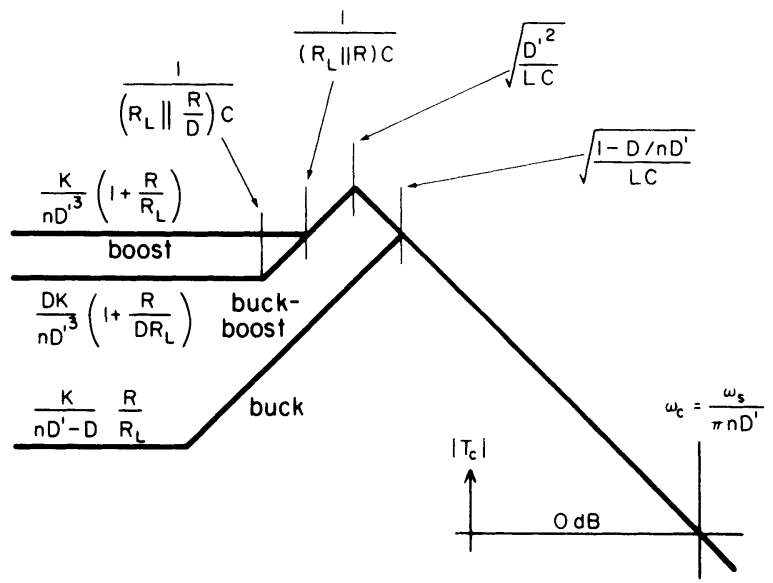

Fig. 16. Magnitude asymptotes of the current loop gain $T_{C}$ for the three converters. All have the same crossover frequency $\omega_{c}=\omega_{s} / \pi n D^{\prime}$.

The most interesting feature is that the highfrequency asymptote is the same for all three converters, and has a crossover frequency $\omega_{c}$ given by

$$
\omega_{c}=\frac{K R}{n D^{\prime} L}=\frac{2}{n D^{\prime} T_{s}}=\frac{\omega_{s}}{\pi n D^{\prime}}
$$

Thus, the current loop gain crosses over on a $-20 \mathrm{~dB} /$ decade slope at a frequency that is a fraction of the switching frequency dependent only upon the duty ratio and not upon any other operating condition or load. If the stabilizing ramp is chosen so that $n=(1+D) / D^{\prime}, \omega_{c}=\omega_{s} / \pi(1+D)$, so the crossover frequency varies at most from about a sixth to a third of the switching frequency. If there is no stabilizing ramp, $\mathrm{n}=1$ and the crossover frequency varies at most from about a third to two-thirds of the switching frequency (if $n=1, D^{\prime}$ cannot be less than 0.5 for stability). In any case, the current loop may be considered wide-band, at least in relation to the bandwidth of the major voltage loop of typical regulators, and also in relation to the validity limit of the state-space average model.

Another feature of interest is the low frequency value of the current loop gain $\mathrm{T}_{c}(0) \equiv$ $\mathrm{T}_{\mathrm{C}}(\mathrm{s}=0)$. For each converter, this value is proportional to $\mathrm{K} \equiv 2 \mathrm{~L} / \mathrm{RT}_{\mathrm{S}}$, which is identical with the "conduction parameter" defined in relation to duty ratio programmed discontinuous conduction mode [7]. Hence, $K$ is a measure of the low-frequency current loop gain $\mathrm{T}_{\mathrm{c}}(0)$ and is a factor in the gain box $\mathrm{F}_{\mathrm{m}}$ in Figs. 4 and 11 through 15 . Physically, high $\mathrm{K}$ (large L) means high current loop gain because the inductor current slope in Fig. 10 is shallower, so that less error between $i_{\ell}$ and the control waveform is needed to provide the required duty ratio. In other words, the inductor current approaches more closely the value demanded by the control signal.

It was shown in [7] that in order to maintain continuous conduction mode, $\mathrm{K}$ must exceed some critical value $\mathrm{K}_{\text {crit }}$, where $\mathrm{K}_{\text {crit }}$ has the respective values $\mathrm{D}^{\prime}, \mathrm{DD}^{\prime 2}$, $\mathrm{D}^{\prime} 2$ for the buck, boost, and buckboost converters. In terms of the appropriate $\mathrm{K}_{\mathrm{crit}}$, the values of $\mathrm{T}_{\mathrm{C}}(0)$ are:

Buck Converter

$$
T_{c}(0)=\frac{K}{K_{\text {crit }}} \frac{D^{\prime}}{n^{\prime}-D} \frac{R}{R_{L}}
$$

Boost Converter

$$
\mathrm{T}_{\mathrm{c}}(0)=\frac{\mathrm{K}}{\mathrm{K}_{\text {crit }}} \frac{\mathrm{D}}{\mathrm{nD}^{\prime}}\left(1+\frac{\mathrm{R}}{\mathrm{R}_{\mathrm{L}}}\right)
$$

Buck-boost Converter

$$
\mathrm{T}_{\mathrm{c}}(0)=\frac{\mathrm{K}}{\mathrm{K}_{\text {crit }}} \frac{\mathrm{D}}{\mathrm{nD}^{\prime}}\left(1+\frac{\mathrm{R}}{\mathrm{DR}_{\mathrm{L}}}\right)
$$

If the stabilizing ramp is chosen such that $\mathrm{n}=$ $(1+D) / D^{\prime}$, and if $R=R_{L}$, these results reduce to

$$
\mathrm{T}_{\mathrm{c}}(0)=\frac{\mathrm{K}}{\mathrm{K}_{\text {crit }}} \mathrm{A}
$$

where $A=D^{\prime}, 2 D /(1+D), 1$ respectively for the buck, boost, and buck-boost converters. In a11 cases, A is of order unity.

Regulator design considerations usually dictate use of the smallest $\mathrm{K}$, which must be greater than $\mathrm{K}_{\mathrm{crit}}$ to avoid dropping into discontinuous conduction. This lower limit occurs at minimum load current, typically 10 percent of maximum load current. Hence, the lowest $\mathrm{T}_{\mathrm{c}}(0)$ is also of order unity, at minimum load current, and may be an order of magnitude greater at maximum load current.

Therefore, the current loop gain may be considered to be low, at least in relation to loop gains typically employed to regulate a given quantity, in this case the inductor current, and is in fact desired to be low in order to keep the inductance value as small as possible.

\subsection{The y Parameter Mode1}

It has been shown in the previous section that the loop gain of a current-programmed power stage is not large, so the quantity being sensed, the inductor current, is not very tightly regulated. Despite the wide bandwidth, crossover occurs on a single-pole slope and so there is no question of stability as long as the conventional stabilizing ramp is employed.

These considerations, plus the fact that the quantity being regulated is not the output current (except for the buck converter), so that two separate blocks $G_{1}$ and $G_{2}$ are required in the model of Fig. 4, suggest that the current feedback loop is not a particularly useful modelling concept, and that the control voltage to output transfer function $\hat{\mathrm{v}} / \hat{\mathrm{v}}_{\mathrm{c}}$ might be just as well modelled by an equivalent 
circuit, representing a "new" power stage as in [3], in which the current feedback is absorbed and not identified as a loop.

These arguments apply with even greater force to modelling the line input to output transfer function $\hat{v} / \hat{v}_{g}$. This is because the line input $\hat{v}_{g}$ affects the output $\hat{v}$ not only directly via the canonical model of Figs. 11 through 13, but also via the "feedforward" path represented by the $G_{6}$ gain box, so that $\hat{v} / \hat{v}_{g}$ is not simply its open-loop value divided by $1+T_{C}$.

Since current-programming tends (a1though poorly) to regulate the inductor current, and there$\ddot{v}_{9}$
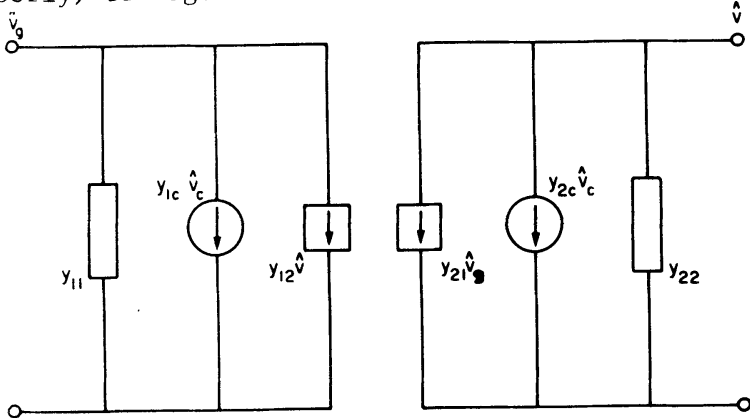

Fig. 17. Model in y parameter form, suitable when input and output currents tend to be constant. Parameter values are given in

Table 1 .

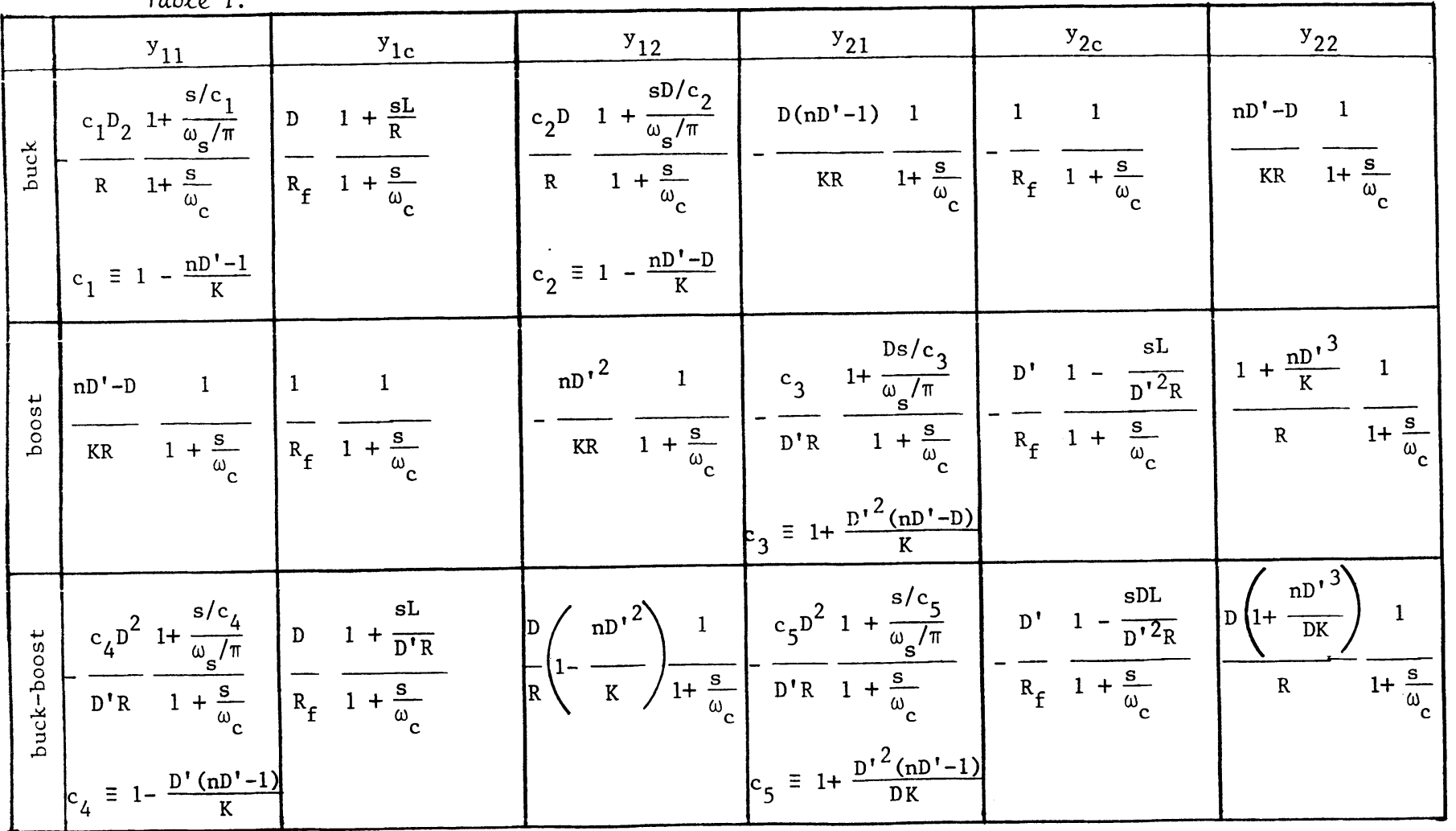

Table 1. Expressions for the $y$ parameters of Fig. 17 for the current-programmed buck, boost, and buck-boost converters: $R \equiv V / I$ is the output operating point parameter; $K=2 L / R T_{S}$ is the conduction parameter; $n \equiv 1+2 M_{C} / M_{1}$ contains the stabilizing ramp slope $M_{C} ; \omega_{s}$ is the switching frequency, and $\omega_{C}=\omega_{S} / \pi n D^{\prime}$ is the current loop gain crossover frequency. fore also the line input and output currents of the power stage, in proportion to the control voltage, a $y$ parameter model is an appropriate first choice. This mode1, shown in Fig. 17, contains six parameters and is sufficient to determine the output voltage and line input current as functions of the control voltage and the line input voltage.

The y parameters can be found for each currentprogrammed converter stage by matching each y parameter of Fig. 17 with the appropriate function calculated from Fig. 11, 12, or 13; for example, $y_{21}$ is the short-circuit output current as a function of the line input voltage with zero control voltage.

The results are collected in Table I. All the c coefficients, which are introduced merely for compactness, are of order unity, and approach unity as $\mathrm{K}$ becomes large.

There are several features of interest exposed in the results of Table $I$. Each y parameter has a single pole at the current loop gain crossover frequency $\omega_{c}=\omega_{s} / \pi n D^{\prime}$ which, as shown in the previous section, may lie between one sixth and twothirds of the switching frequency $\omega_{s}$. Some of the $y$ parameters have zeros of order $\omega_{s} / \pi$, or one-third the switching frequency. If the poles and zeros in $\omega_{c}$ and $\omega_{s} / \pi$ are ignored, and if the stabilizing ramp is such that $\mathrm{n}=(1+\mathrm{D}) / \mathrm{D}^{\prime}\left(M_{\mathrm{C}}=M_{2}\right)$, the y parameter results reduce to those of Fig. 17 in [3], which further reduce to those of Fig. 14 in [3] if $\mathrm{K}$ is 
sufficiently large. These "simple" results correspond to large current loop gain which, as shown in the previous section, is unlikely to occur in a realistic regulator circuit.

Apart from the control parameters $y_{1 c}$ and $y_{2 c}$, the other four y parameters are all inversely proportional to the output operating point parameter $\mathrm{R} \equiv \mathrm{V} / \mathrm{I}$. Some of them are inversely proportional to $\mathrm{KR}$, which means that they vanish in the infinite current loop gain limit. This happens for $\mathrm{y}_{21}$ and $\mathrm{y}_{22}$ for the buck converter, which results from the fact that it is the output current that is being sensed and so the output impedance should approach infinity (current source) in the infinite current loop gain limit. This also happens for $\mathrm{y}_{11}$ and $\mathrm{y}_{12}$ for the boost converter, because it is the input current that is being regulated. On the other hand, none of the $y$ parameters vanishes in the infinite current loop gain limit for the buck-boost converter, because the sensed current is neither the input nor the output current.

\section{FURTHER ASPECTS OF THE CURRENT-PROGRAMMED MODEL}

Even though considerable information can be gained from the y parameter model established in the previous section, it may be useful to investigate other possible forms of the model. One potentially useful form is that of the original canonical model for duty ratio programmed continuous conduction mode; perhaps some insight can be gained into how the current programming causes a divergence of the element values from those in this model.

This possibility is pursued in Section 5.1, at least as far as representation of the control-tooutput properties. One useful result that emerges is that the control to output voltage transfer function actually has two poles, instead of the single one that is normally associated with currentmode programming. This two-pole function is developed in Section 5.2.

\subsection{Manipulation into the Duty Ratio Programmed Format}

To manipulate the output half of the y parameter model of Fig. 17 into a form resembling the original canonical model, the Norton form of $y_{2 c}$ and $y_{22}$ is changed into the Thevenin equivalent, and the conversion transformer $1: M$ is introduced, as shown in Fig. 18, where $M$ is the conversion ratio.

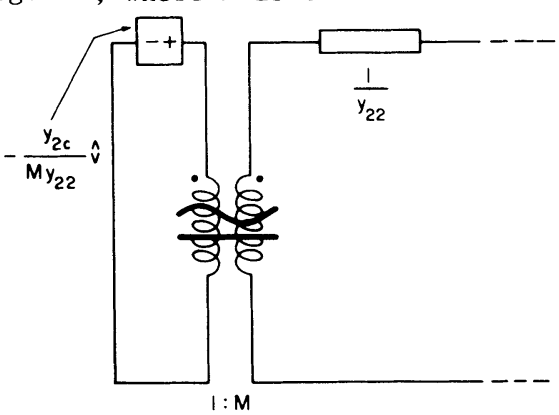

Fig. 18. Manipulation of the $y_{2 c}$ and $y_{22}$ parameters into a Thevenin equivalent.
Evaluation of the effective voltage modulation generator and the effective series impedance of Fig. 18 shows that for each converter the series impedance is the sum of a resistance and an inductance, and the generator has an rhp zero in the case of the boost and buck-boost converters. Thus, this format does resemble the original canonical model.

The boost converter will be taken as an example. The results are:

$$
\begin{aligned}
& -\frac{\mathrm{y}_{2 c}}{\mathrm{My}_{22}}=\frac{\mathrm{D}^{\prime 2} \mathrm{R}}{\mathrm{R}_{\mathrm{f}}\left(1+\mathrm{nD}^{\left.\prime^{3} / \mathrm{K}\right)}\right.}\left(1-\frac{\mathrm{sL}}{\mathrm{D}^{\prime 2} \mathrm{R}}\right) \\
& \frac{1}{\mathrm{y}_{22}}=\mathrm{R} \frac{\mathrm{K} / \mathrm{nD}^{\prime 3}}{1+\mathrm{K} / \mathrm{nD}^{\prime^{3}}}+\mathrm{s} \frac{\mathrm{L}}{\mathrm{D}^{\prime 2}} \frac{1}{1+\mathrm{K} / \mathrm{nD}^{\prime^{3}}}
\end{aligned}
$$

The term $\mathrm{K} / \mathrm{nD}^{\prime 3} \equiv \mathrm{T}_{\mathrm{C} \infty}$ can be identified from Eq. (30) as the low frequency current loop gain when $\mathrm{R}_{\mathrm{L}}=\infty$, so that the above results can be written

$$
\begin{aligned}
& -\frac{y_{2 c}}{M_{22}}=\frac{D^{\prime 2} R}{R_{f}} \frac{T_{C \infty}}{1+T_{C \infty}}\left(1-\frac{s L}{D^{\prime 2} R}\right) \\
& \frac{1}{y_{22}}=R \frac{T_{C \infty}}{1+T_{C \infty}}+s \frac{L}{D^{\prime 2}} \frac{1}{1+T_{C \infty}}
\end{aligned}
$$

The complete model of the current-programmed boost converter can therefore be represented as in Fig. 19. This format clearly shows that the output

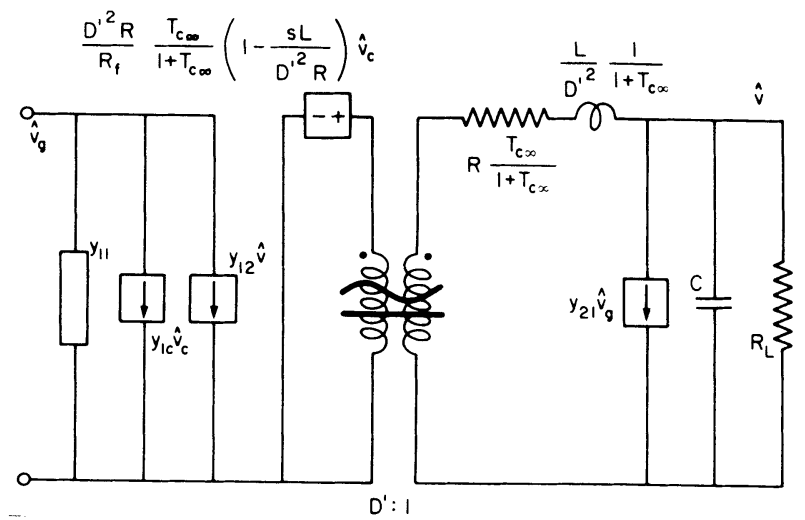

Fig. 19. Model of the current-programmed boost converter with the output part in the same format as the duty ratio programmed model.

LC filter remains, as in the canonical model for the duty ratio programmed mode; however, the values are different. Current-mode programming introduces a series damping resistance $\mathrm{RT}_{\mathrm{co}} /\left(1+\mathrm{T}_{\mathrm{co}}\right)$, which is what makes the output resemble a current source rather than a voltage source and gives the familiar dominant-pole response with the load $C$ and $R_{L}$. Notice, however, that the inductance does not vanish except in the infinite loop gain limit (which is unlikely to be approached), and so there remains a second pole at a higher frequency in the control to output voltage transfer function. Therefore, current-programming can be considered to increase 
substantially the damping of the converter LC filter, which separates the two poles and makes the lower one dominant.

This damping effect is lossless, in the same sense as that produced by storage-time modulation, described in [8]. In fact, both arise from effects that are the same in principle: storage-time modulation causes the effective switch duty ratio to be affected internally by the switch current; currentprogramming is a more extreme case of the same effect in which the switch current (which is the same as the inductor current) is externally and purposely caused to determine the duty ratio.

Manipulation of the input model in Fig. 19 could also be undertaken, in order to make the complete model resemble more closely the duty ratio programmed canonical model. Although this can be achieved, the element values are rather complicated, and do not seem to give much additional insight into the performance. This probably results from the inherently complicated way in which the line input $\hat{v}_{g}$ affects the input current and the output voltage, as indicated by the requirement for four gain boxes connected to $\mathrm{vg}_{\mathrm{g}}$ in Fig. 4 .

The principal value of the model of Fig. 19 is the exposure of the nonvanishing filter inductance and the consequent retention of the second pole in the control to output transfer function. Even so, the quantitative relation is more easily derived from the original y parameter model of Fig. 17.

\subsection{The Control-to-Output Transfer Function $\hat{v} / \hat{v}_{c}$}

For the purpose of determining the control to output voltage transfer function $\hat{v} / \hat{v}_{c}$, the relevant part of Fig. 17 can be presented as in Fig. 20, with the immittances shown explicitly in factored polezero form. The low-frequency values $R_{2 c}$ and $R_{22}$ can be identified from Table $I$, as can the rhp zero $\omega_{z}$ in the case of the boost and buck-boost. The current loop gain crossover frequency is $\omega_{c}=$ $\omega_{S} / \pi n D^{\prime}$, and the load corner frequency is $\omega_{L} \equiv 1 / R_{L} C$.

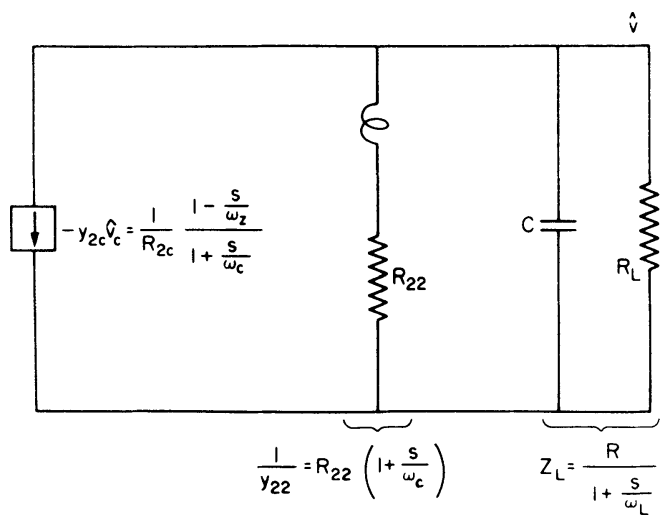

Fig. 20. Output part of the $y$ parameter model explicitly showing the poles and zeros.
Straightforward analysis leads to

$\hat{v}_{c}=\frac{R_{22} \| R_{L}}{R_{2 c}} \frac{1-\frac{s}{\omega_{z}}}{\left(1+\frac{s}{\left(1+R_{L} / R_{22}\right)\left(\omega_{L} \| \omega_{c}\right)}\right)\left(1+\frac{s}{\omega_{L}+\omega_{c}}\right)}$

subject only to the approximation that the two poles are well separated. Since usually $\omega_{\mathrm{L}} \ll \omega_{\mathrm{C}}$, this is justified and the result can be further approximated to

$\frac{\hat{v}}{\hat{v}_{c}}=\frac{R_{22} \| R_{L}}{R_{2 c}} \frac{1-\frac{s}{\omega_{z}}}{\left(1+\frac{s}{1 /\left(R_{22} \prod R_{L}\right) c}\right)\left(1+\frac{s}{\omega_{c}}\right)}$

It may be noted that this result corresponds to neglect of the inductance in Fig. 20; the dominant pole is then immediately $1 /\left(R_{22}|| R_{L}\right) C$, and the second pole and rhp zero come directly from y2c. The asymptote graph of Eq. (51) is shown in Fig. 21.

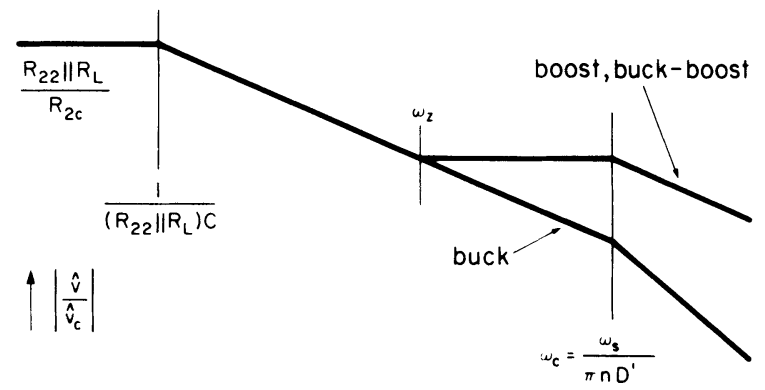

Fig. 21. Magnitude asymptotes of the control-tooutput transfer function $\hat{v} / \hat{v}_{\mathrm{C}}$ for the three basic converters. In addition to the dominant pole, there is a second pole at the current loop gain crossover frequency $\omega_{C}=\omega_{S} / \pi n D^{\prime}$.

Since the second pole at the current loop gain crossover frequency $\omega_{c}$ may occur at as low as a sixth of the switching frequency $\omega_{s}$, its presence may significantly affect the major voltage loop gain phase margin in a complete regulated power supply, especially with the additional phase lag from the rhp zero in a boost or buck-boost regulator.

\section{CONCLUSIONS}

If any value is to be extracted from the concept of a feedback loop, loops thus identified should include those that are purposely established in order to regulate some particular quantity. For example, in the familiar voltage regulator one defines an overall feedback loop whose bandwidth and phase and gain margins define the useful purposes of the system such as its stability and output impedance. 
This approach is not negated even if there is an internal "minor" loop inside the overall "major" loop, which is established, for example, by the common practice of incorporating local feedback around the error amplifier. This "nested" minor loop is treated by itself: its stability is established (although this is rarely in question), and its closed-loop properties are obtained. Henceforth, it is simply incorporated as a gain block in the major loop gain, and this treatment is entirely consistent with the design-oriented analysis approach in which the closed-loop properties of the error amplifier constitute the frequency compensation characteristic to shape the major loop gain.

A current-programmed loop around the power stage of a pwm voltage regulator is exactly the same in principle: its purpose is to regulate the inductor current and, among other things, to shape the major loop gain characteristic. These objectives are kept clearly in mind by modelling the current-programmed power stage independently of the major loop, and then incorporating its closed-loop properties, modelled as a "new" power stage, as a gain block in the major loop gain. There is no more value in considering the current-programming minor feedback path in paralle1 with the major voltage feedback path, thus determining the "total" feedback around the power stage, than there is in considering the error amplifier minor feedback path in parallel with the major voltage feedback path.

These conclusions are reached in Section 2, and justify the objective of modelling the currentprogrammed power stage separately from the major voltage loop. This is done in Sections 3 and 4 , in which the question is considered as to whether or not it is useful to emphasize the feedback loop characteristics. To do this, a new model is established in which the current-programmed loop is characterized by appropriate gain boxes that augment the we11-known model for the duty ratio programmed power stage in continuous conduction mode. The three versions of this model, for the buck, boost, and buck-boost converters, are shown in Figs. 11 through 13 .

A current loop gain $\mathrm{T}_{\mathrm{C}}$ is found from each of these models, in Section 4.1. The loop gain crosses over on a $-20 \mathrm{~dB}$ slope at the same frequency $\omega_{c}$ for a11 three converters, where $\omega_{c}$ depends only upon the switch duty ratio and the stabilizing ramp, and lies between one-sixth and two-thirds of the switching frequency $\omega_{\mathbf{S}}$. Despite the relatively wide bandwidth, the low-frequency loop gain is low, at least in typical designs in which the power stage inductor is chosen to have the smallest value consistent with maintenance of the continuous conduction mode at minimum load.

Additional features of the current-programmed loop are that the line to output transfer function is complicated, because the line voltage affects the operation in several ways. Also, the quantity being regulated, the inductor current, is not the output current of the power stage except in the case of the buck converter.
A11 these considerations suggest that description of the current-programmed power stage in terms of its feedback loop properties is not particularly enlightening, and therefore in Section 4.2 the current loop is absorbed into a y parameter model, Fig. 17, that represents the effective "new" power stage. The expressions for the y parameters are assembled in Table $\mathrm{I}$, and are extended versions of the results previously obtained in [3]. The extensions consist of retention of an arbitrary stabilizing ramp slope contained in the parameter $\mathrm{n}$, and retention of poles and zeros of order $\omega_{\mathrm{c}}$ and $\omega_{s} / \pi$. The presence of the poles at $\omega_{c}$ represents the only visible connection with the current loop gain, whose crossover frequency is $\omega_{c}$.

In Section 5.1 some manipulation of the current-programmed model leads to a form resembling the original duty ratio programmed model, in which the output LC filter is explicit. It is seen that current-programming introduces a substantial series damping resistance and also reduces the effective value of the inductance. The damping resistance is lossless, since it arises from a feedback effect, in exactly the same way as a corresponding lossless resistance arises due to switch storage-time modulation.

This form of the model shows how currentprogramming tends to make the power stage output behave like a current source; however, since the effective inductance, although reduced, dnes not vanish, there remains a second pole well beyond the familiar dominant pole that results from the current source feeding the load resistance and output capacitance.

The actual control to output voltage transfer function is derived in Section 5.2, where it is shown that the second pole is at the current loop gain crossover frequency $\omega_{c}$. The presence of this second pole could have a significant effect on the voltage major loop stability margins, especially for boost and buck-boost power stages because of the additional phase lag due to the right halfplane zero. 


\section{REFERENCES}

[1] Cecil W. Deisch, "Simple Switching Contro1 Method Changes Power Converter into a Current Source," IEEE Power Electronics Specialists Conference, 1978 Record, pp. $300-306$ (IEEE Publication 78CH1337-5 AES).

[2] A. Cape1, G. Ferrante, D. O'Sullivan, and A. Weinberg, "Application of the Injected Current Model for the Dynamic Analysis of Switching Regulators with the New Concept of LC ${ }^{3}$ Modulator," IEEE Power Electronics Specialists

Conference, 1978 Record, pp. 135 - 147 (IEEE Pub1ication 78CH1337-5 AES).

[3] Shi-Ping Hsu, Art Brown, Loman Rensink, and R. D. Middlebrook, "Modelling and Analysis of Switching Dc-to-Dc Converters in ConstantFrequency Current-Programmed Mode," IEEE Power Electronics Specialists Conference, 1979 Record, pp. 284 - 301 (IEEE Publication 79CH1461-3 AES).

[4] B. H. Cho and F. C. Lee, "Measurement of Loop Gain with the Digital Modulator," IEEE Power Electronics Specialists Conference, 1984 Record, pp. 363 - 373 (IEEE Publication $84 \mathrm{CH} 2000-8$ ).

[5] G. W. Wester and R. D. Middlebrook, "LowFrequency Characterization of Switched Dc-toDc Converters," IEEE Power Processing and Electronics Specialists Conference, 1972 Record, pp. 9 - 20 (IEEE Publication 72CH0652-8 AES); also IEEF Trans. Aerospace and Electronic Systems, vol. AES-9, pp. 376 - 385, May 1973.
[6] R. D. Middlebrook and Slobodan Cuk, "A General Unified Approach to Modelling Switching Converter Power Stages," IEEE Power Electronics Specialists Conference, 1976 Record, pp. $18-34$ (IEEE Publication 76CH1084-3 AES); also International J. of Electronics, vol. 42, no. 6 , pp. 521 - 550, June 1977.

[7] Slobodan Ćuk and R. D. Middlebrook, "A General Unified Approach to Modelling Switching Dc-toDc Converters in Discontinuous Conduction Mode," IEEE Power Electronics Specialists Conference, 1977 Record, pp. 36 - 57 (IEEE Publication 77CH1213-8 AES).

[8] W. M. Polivka, P. R. K. Chetty, and R. D. Middlebrook, "State-Space Average Modelling of Converters with Parasitics and Storage-Time Modulation," IEEE Power Electronics Specialists Conference, 1980 Record, pp. $119-143$ (IEEE Publication 80CH1529-7).

[9] Hendrik W. Bode, Network Analysis and Feedback Amplifier Design, Van Nostrand, 1945.

[10] John J. D'Azzo and Constantine H. Houpis, Feedback Control System Analysis and Synthesis, McGraw-Hi11, 1960.

[11] Hendrik W. Bode, op.cit., p.10.

[12] John L. Bower and Peter Schultheiss, Introduction to the Design of Servomechanisms, John Wiley \& Sons, 1958; section 5.6. 